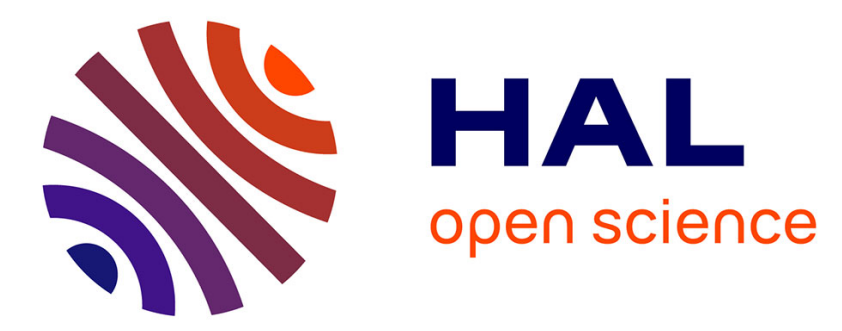

\title{
Extension of the partially integrated transport modeling method to the simulation of passive scalar turbulent fluctuations at various Prandtl numbers
}

Bruno Chaouat, Roland Schiestel

\section{- To cite this version:}

Bruno Chaouat, Roland Schiestel. Extension of the partially integrated transport modeling method to the simulation of passive scalar turbulent fluctuations at various Prandtl numbers. International Journal of Heat and Fluid Flow, 2021, 89, pp.108813. 10.1016/j.ijheatfluidflow.2021.108813 . hal03217987

\section{HAL Id: hal-03217987 \\ https://hal.science/hal-03217987}

Submitted on 5 May 2021

HAL is a multi-disciplinary open access archive for the deposit and dissemination of scientific research documents, whether they are published or not. The documents may come from teaching and research institutions in France or abroad, or from public or private research centers.
L'archive ouverte pluridisciplinaire HAL, est destinée au dépôt et à la diffusion de documents scientifiques de niveau recherche, publiés ou non, émanant des établissements d'enseignement et de recherche français ou étrangers, des laboratoires publics ou privés. 


\title{
Extension of the Partially Integrated Transport Modeling method to the simulation of passive scalar turbulent fluctuations at various Prandtl numbers
}

\author{
Bruno Chaouat ONERA, Université Paris-Saclay, 92322 Châtillon, France \\ Roland Schiestel IRPHE/CNRS, 13384 Marseille, France
}

March 31, 2021

\begin{abstract}
The present work aims to extend the hybrid non zonal RANS/LES partially integrated transport modeling (PITM) method to turbulent flows in the presence of passive scalar contaminant for simulating large scales of turbulent flows. Focussing on the methodological aspects, we derive the basic transport equations both for the scalar variance of fluctuations and dissipation-rate of the variance. The basis of the method was introduced in references [R. Schiestel and A. Dejoan, "Towards a new partially integrated transport model for coarse grid and unsteady turbulent flow simulations ", Theor. Comput. Fluid Dyn. 18, 443 (2005)] and [B. Chaouat and R. Schiestel, "A new partially integrated transport model for subgrid-scale stresses and dissipation rate for turbulent developing flows ", Phys. Fluids 17, 065106 (2005)]. It provides a continuous approach for hybrid Reynolds averaged Navier-Stokes equations-large eddy simulation (RANS-LES) with seamless coupling between RANS and LES regions. The main motivation is to simulate accurately in LES mode performed on coarse grids, scalar fluctuation fields, in addition to mean scalar fields. As known, the knowledge of the rms scalar fluctuations is often involved in handling practical engineering and geophysical flows. Like in dynamical equations, it is found that the coefficient appearing in the destruction term of the dissipation-rate of the scalar variance is a function of the cutoff-wave number and also of the Prandtl number and the Reynolds number. Depending on the
\end{abstract}


value of the Prandtl-number, different expressions of this function have been derived according to the relevant physics in the wave number space. Finally, numerical simulations of fully turbulent flows including passive scalar transport fields have been performed on several meshes of medium and coarse grid resolutions at the Reynolds number $R_{\tau}=395$ for the Prandtl numbers $P_{r}=0.1$, 1 and 10, respectively associated with heat transfer of liquid metals, gas and water for illustrating the theoretical development made on PITM. As expected, the PITM method provides satisfactory results in good agreement with data of direct numerical simulations. From a general point of view, this work opens new routes of modeling and simulation of turbulent flows including a passive scalar with a drastic reduction of the computational time and memory in term of number of grid points in comparison with the demanding resources of highly resolved LES.

\section{Introduction}

Mathematical turbulence modeling methods such as Reynolds averaged Navier-Stokes (RANS) or large eddy simulations (LES) methods have been proposed independently from each other for simulating turbulent flows. Each of them has their respective advantage and limitation and their specific field of application so that they should be considered as complementary tools in computational fluid dynamics. In that sense, the most appropriate tool does not depend on the intrinsic performances of the method itself but more precisely on both the required computational ressource and nature of the flow. RANS models have been developed initially for simulating flows that evolve slowly in time with a reasonable computational cost and are rather used in industry for determining practical data such as the pressure and heat coefficients used in the optimisation of engineering processes while LES demanding large computational cost is rather used for simulating unsteady flows subjected to high frequencies and are often studied in research laboratories with emphasis on fundamental aspects and the turbulence structures. Since the past two-decade, hybrid RANS-LES have been proposed to take benefit of both RANS and LES methods (Fröhlich and Von Terzi, 2008, Chaouat, 2017). Among these numerous hybrid RANS/LES methods, the partially integrated transport modeling (PITM) is an advanced method that has been developed in previous publications (Schiestel and Dejoan, 2005; Chaouat and Schiestel, 2005, 2009, 2012, 2013; Chaouat, 2010, 2012, 2017b) for simulating large eddy scale of turbulent flows out of spectral equilibrium performed on coarse grids with a mesh step corresponding to a spectral 
cutoff wave number that may be located before the inertial zone of the Kolmogorov range. This type of approach allows continuous hybrid non-zonal simulations between near RANS regions and LES regions with seamless coupling. The PITM model reduces to RANS when the grid step increases, if the flow is homogeneous in the three directions. Depending on the value of the spectral splitting parameter, usually linked to the numerical mesh, each part of the fluctuating turbulent flow is partially modeled and partially simulated like in the LES method. There is a unique link between the filter width or the splitting wavenumber and the turbulent energy ratio of subfilter energy to simulated energy. The PITM models were the first to introduce this practice (Schiestel and Dejoan, 2005; Chaouat and Schiestel, 2005) and some other models of the literature, like the partially averaged Navier-Stokes (PANS) models recently borrowed this formulation in order to improve their formalism (Foroutan and Yavuzkurt, 2014). The method is originally developed on a spectral basis, but recently a variational analysis has been conducted to handle inhomogeneous flows (Heinz, 2019). The main feature of this method lies in its general character that allows to convert almost any usual one point RANS transport closure into a corresponding hybrid non-zonal subfilter-scale model for performing large eddy scale simulations. So, PITM can be applied to two-equation turbulence models but also to more advanced turbulence models such as Reynolds stress models developed in the framework of second moment closure (SMC) (Schiestel, 2007; Hanjalic and Launder, 2011).

The present work deals with the extension of the PITM method to passive scalar fluctuations. We shall give emphasis in the theoretical development of the methodology and derivation of equations applied to scalar variance from a physical standpoint. Applications will be restricted here to the case of the plane channel flow with associated heat transfer in order to demonstrate the satisfactory behavior of the model. More extensive and varied applications will be considered in subsequent further works. This work is highly motivated by the fact that passive scalar fields are often encountered both in nature including geophysical and environmental flows as well as engineering flows in industrial processes. In RANS and LES simulations involving scalar fields for temperature or concentration, it is of primary importance to accurately model the fluxes of the scalar-velocity correlations that play a major role in the determination in heat and mass transfer in turbulent flows. Indeed the mean scalar field equations are directly coupled with the turbulence dynamic equations. But, the knowledge of the rms fluctuations 
of the scalar field may also be of importance in several practical cases when the high scalar fluctuations can impact the wall structures. Structures exposed to such temperature fluctuations may suffer from thermal fatigue damage. This problem may appear in the mixing of two flows at different temperatures merging at a T-junction. For instance, among others, thermal striping can arise in certain liquid metal-cooled reactors. Because this may result in thermal fatigue cracking, the problem deserves study. In the present work, we shall give a special focus to the study of the variance of fluctuations of the scalar, keeping in mind that scalar transfer problems are inherently interrelated. The same general form for the turbulent scalar equations invites us to consider any kind of scalar. Overall, among the large variety of passive scalar, we can for instance point out the temperature when it is possible to neglect the dynamic effect of density variations but in a more general way, it can be also any passive contaminant concentration transported by the flow itself that deserves physical investigations.

After briefly recalling the basics of the PITM method, we will extend it to the study of scalar contaminant fluctuations. In the present case according to previous works of the authors Chaouat and Schiestel $(2005,2009,2012,2013)$ and Chaouat $(2010,2012,2017)$, we will retain the second moment closure for modeling the subfilter scale stresses considering that this is the appropriate level of closure to get accurate results of complex turbulent flow fields. However a first order closure based on diffusivity models will be sufficient in a first step for modeling the scalar fluxes involved in the production of variance equation for the subfilter passive scalar (Schiestel, 2007; Hanjalic and Launder, 2011). There are several arguments in favor of this choice. Firstly, the turbulent velocity field is here decoupled from the passive scalar field. Secondly, tractable equations can be solved easily from an engineering point of view in both research and engineering CFD codes. Hence, this strategy to choose different levels of closure for each type of flow field is justified here both from physical and numerical points of view while allowing fair results for the scalar field. In this work, we will give emphasis on the PITM extension to scalar transport that comes from the new theoretical developments in the dissipation rate of variance equation. As this equation is common both to first order and higher order closure, it would be a simple matter to extend the present work to second order passive scalar closure. 


\section{The basics of the PITM method}

\subsection{General framework : the levels of description}

The PITM method is funded originally on the spectral equations for quasi-homogeneous turbulence. With an hypothesis of tangent homogeneous space (Chaouat and Schiestel, 2007) it becomes then possible to deal with non-homogeneous flows with a good approximation for practice. The steps used in the development of the model introduce several level of description which are made clear in the synoptic (Table 1) for dynamic and scalar turbulent quantities. In shear driven turbulent flows in the presence of a passive scalar, the transport equation for the turbulent stresses and the turbulent scalar fluxes $\Phi_{i j}$ and $\Phi_{i \theta}$, respectively, appearing in lines 3 and 4 of table 1 are the most important tensors in second order closures to describe turbulent transfer. These equations, at all level of description, contain important redistribution terms linked to pressure fluctuations that have been a challenge in spectral closures and in RANS closures as well. In the turbulent stresses closure, the redistribution term of turbulence energy is usually modeled using several contributions including a linear contribution (rapid part involving the mean velocity gradients) and a non-linear contribution (slow part or Rotta term) along with a wall contribution. In the equation for the scalar fluxes, a similar approach can be used, introducing the corresponding three contributions. The study of these term in the spectral context has been extensively developed (Cambon et al., 1981). If the linear terms are closed at level 1, a parametrization of angular dependence with the wave vector $\boldsymbol{\kappa}$ is necessary at level 2 . As a consequence of spherical averaging, a closure for linear terms is required like in RANS models. A more advanced representation of the linear terms is worked out (Mons et al., 2016) by introducing two anisotropy tensors for the polarization anisotropy and for the directional anisotropy in wave vector, while the non-linear transfer is obtained from eddy damped quasi-normal Markovian (EDQNM) hypothesis. This spectral modeling approach has been extended also to passive scalar transport (Briard et al., 2016). In this model, the equations for temperature variance as well as the turbulent thermal fluxes have been closed using directional and polarization parameters in homogeneous anisotropic fields. For the subfilter equations (level 3) the pressure-strain closures are inspired from RANS models and will be used in this paper both for the slow and rapid parts. So, in the present simulations, the subfilter stress tensor $\left(\tau_{i j}\right)_{s f s}$ is numerically calculated from its transport equation considering the turbulence 
Table 1: Different levels of description in turbulence modeling including a passive scalar

\begin{tabular}{|l|l|l|l|l|}
\hline Turbulence quantity & $\begin{array}{l}\text { Level 1 } \\
\text { 3D spectrum }\end{array}$ & $\begin{array}{l}\text { Level 2 } \\
\text { Spherical av- } \\
\text { eraged spec- } \\
\text { trum }\end{array}$ & $\begin{array}{l}\text { Level 3 } \\
\text { Subfilter scale } \\
\text { part }\end{array}$ & $\begin{array}{l}\text { Level 4 } \\
\text { Mean subfil- } \\
\text { ter scale part }\end{array}$ \\
\hline $\begin{array}{l}\text { Variance of velocity } \\
\text { fluctuations }\end{array}$ & $\mathcal{E}(\boldsymbol{X}, \boldsymbol{\kappa}, t)$ & $E(\boldsymbol{X}, \kappa, t)$ & $k_{s f s}(\boldsymbol{X}, t)$ & $\left\langle k_{s f s}\right\rangle(\boldsymbol{X})$ \\
\hline $\begin{array}{l}\text { Variance of passive } \\
\text { scalar fluctuations }\end{array}$ & $\mathcal{E}_{\theta}(\boldsymbol{X}, \boldsymbol{\kappa}, t)$ & $E_{\theta}(\boldsymbol{X}, \kappa, t)$ & $k_{\theta s f s}(\boldsymbol{X}, t)$ & $\left\langle k_{\theta s f s}\right\rangle(\boldsymbol{X})$ \\
\hline $\begin{array}{l}\text { Double velocity corre- } \\
\text { lations }\end{array}$ & $\Phi_{i j}(\boldsymbol{X}, \boldsymbol{\kappa}, t)$ & $\varphi_{i j}(\boldsymbol{X}, \kappa, t)$ & $\left(\tau_{i j}\right)_{s f s}(\boldsymbol{X}, t)$ & $\left\langle\left(\tau_{i j}\right)_{s f s}\right\rangle(\boldsymbol{X})$ \\
\hline $\begin{array}{l}\text { Velocity passive scalar } \\
\text { correlations }\end{array}$ & $\Phi_{i \theta}(\boldsymbol{X}, \boldsymbol{\kappa}, t)$ & $\varphi_{i \theta}(\boldsymbol{X}, \kappa, t)$ & $\left(\tau_{i \theta}\right)_{s f s}(\boldsymbol{X}, t)$ & $\left\langle\left(\tau_{i \theta}\right)_{s f s}\right\rangle(\boldsymbol{X})$ \\
\hline
\end{tabular}

spectral tensor $\varphi_{i j}$. The same type of terms appear in the equation for the subfilter scalar fluxes $\left(\tau_{i \theta}\right)_{s f s}$ that will be considered subsequently using the turbulence scalar spectral vector fluxes $\varphi_{i \theta}$. It is worth noting that in passive scalar transport, the scalar variance $k_{\theta}$ as well as its dissipation rate $\epsilon_{\theta}$ are not at all involved in the dynamic equations. But the reverse is not true, the equations for these quantities are dependent on many other turbulent correlations including the scalar-velocity correlations in addition to the dynamical correlations. In particular, the production term in the scalar variance equation involves the aforementioned scalar-velocity correlation tensor corresponding physically to the turbulent scalar fluxes $\varphi_{i \theta}$. A modeled transport equation for these fluxes is of utmost importance in the study of turbulent scalar transport and would be required here to stick to the second order of closure. However, considering that our present focus is mainly devoted to the study of scalar fluctuations, we shall postpone this requirement to a further study. We will retain here a gradient approximation of this term using a tensorial diffusivity. The main concern of the present paper is therefore to extend the spectral methods already developed by the present authors in dynamic turbulence to the fluctuations of the transported scalar. 


\subsection{Spectrum splitting and partial integration}

In a general way, each flow variable $\phi$ can be decomposed into a statistical mean value $\langle\phi\rangle$ and a fluctuating turbulent part $\phi^{\prime}$ which is subdivided itself into two ranks of fluctuating parts, a large scale part and a small scale part (Schiestel, 1983a, 1983b, 1987; Chaouat and Schiestel, 2007). This decomposition can be written as $\phi=\langle\phi\rangle+\phi^{<}+\phi^{>}$where $\phi^{<}$and $\phi^{>}$can be defined by the spectral operators

$$
\phi^{<}(\boldsymbol{\xi})=\int_{0<|\boldsymbol{\kappa}|<\kappa_{c}} \widehat{\phi^{\prime}}(\boldsymbol{\kappa}) \exp (j \boldsymbol{\kappa} \boldsymbol{\xi}) d \boldsymbol{\kappa}
$$

and

$$
\phi^{>}(\boldsymbol{\xi})=\int_{\kappa_{c}<|\boldsymbol{\kappa}|<\infty} \widehat{\phi^{\prime}}(\boldsymbol{\kappa}) \exp (j \boldsymbol{\kappa} \boldsymbol{\xi}) d \boldsymbol{\kappa}
$$

in which $\kappa_{c}$, here, denotes the first splitting wave number or spectral cutoff. From a physical standpoint, the PITM method finds its basic foundation in the spectral space of wave numbers considering the production, transfer and dissipation processes of energy acting in each spectral wave number range of the spectrum. Different steps must be undertaken to get the basic equations that constitute the cornerstone of the PITM method. In the first step, for any turbulent variable, we develop the transport equation for the two-point fluctuating correlations in the physical space using the concept of the tangent homogeneous space (Chaouat and Schiestel, 2007, Chaouat, 2017) accounting for the midway position $\boldsymbol{X}=\left(\boldsymbol{x}_{A}+\boldsymbol{x}_{B}\right) / 2$ and the vector difference $\boldsymbol{\xi}=\boldsymbol{x}_{B}-\boldsymbol{x}_{A}$ between the two points A and B. In the second step, we take the Fourier transform of this equation. In the third step, we apply the spherical mean operator (Cambon et al., 1981; Schiestel, 1987; Chaouat and Schiestel, 2007) to get an equation that depends only on the scalar wave number and not anymore on the vector wave number itself. In the last step, we finally return to the physical space from partial integration of the spectrum in the wave number ranges $\left[\kappa_{c}, \kappa_{d}\right]$ to get the corresponding transport equation for the correlation of the fluctuating variable $\left\langle\phi^{>} \phi^{>}\right\rangle$in the physical space. This mathematical physics formalism will be followed in the present work to derive equations for the subfilter scale of the passive scalar variance, as already performed for the dynamics of the flow field (Schiestel, 1987; Schiestel and Dejoan, 2005; Chaouat and Schiestel, 2005, 2007, 2009) by using the shape of spectra as studied by Batchelor (1959) and reinterpreted recently by Warhaft (2000). 


\subsection{Recalling of turbulent velocity field equations}

This section recalls the main concepts of the PITM method that allows to derive the major governing equations for the velocity field. Indeed this is not useless because the extension to scalar transport has to rely upon the dynamic equations. The general approach is founded on the technique of partial spectral integration. First consider splitting the turbulence energy spectrum as sketched in Fig. 1 by introducing a cut-off wavenumber. It can be viewed as a filtering operator in spectral space. We suppose for the present purpose that turbulence is fully developed at high Reynolds number and we will consider low Reynolds number modifications later on. As usually, the Reynolds stress tensor is defined

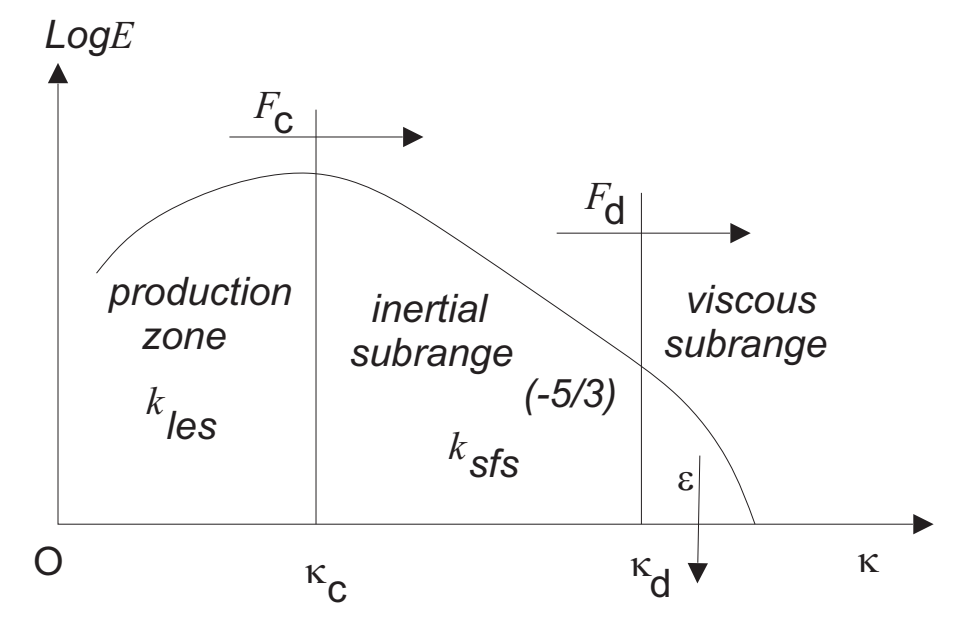

Figure 1: Sketch of spectral splitting of kinetic energy of turbulence

by

$$
\tau_{i j}(\boldsymbol{X}, t)=\left\langle u_{i}^{\prime} u_{j}^{\prime}(\boldsymbol{X}, t)\right\rangle
$$

where $u_{i}^{\prime}$ denotes the usual fluctuating velocity appearing in the statistical treatment and $\boldsymbol{X}$ denotes the midway-position between the two points and $t$ is the time. The starting point is the transport equation of the spherical mean of the Fourier transform of the two-point correlation tensor of the fluctuating velocities denoted $\varphi_{i j}(\boldsymbol{X}, \kappa, t)=\left\langle u_{i}^{\prime} u_{j}^{\prime}(\boldsymbol{X})\right\rangle^{\Delta}(\kappa, t)$ as follows (Hinze, 1975; Schiestel, 1987; Chaouat 
and Schiestel, 2007)

$$
\begin{aligned}
& \frac{\partial \varphi_{i j}(\boldsymbol{X}, \kappa, t)}{\partial t}+\left\langle u_{j}\right\rangle(\boldsymbol{X}) \frac{\partial \varphi_{i j}(\boldsymbol{X}, \kappa, t)}{\partial X_{j}}=\mathcal{P}_{i j}(\boldsymbol{X}, \kappa, t) \\
& +\mathcal{T}_{i j}(\boldsymbol{X}, \kappa, t)+\Psi_{i j}(\boldsymbol{X}, \kappa, t)+\mathcal{J}_{i j}(\boldsymbol{X}, \kappa, t)-\mathcal{E}_{i j}(\boldsymbol{X}, \kappa, t)
\end{aligned}
$$

where $\mathcal{P}_{i j}, \mathcal{T}_{i j}, \Psi_{i j}, \mathcal{J}_{i j}$, and $\mathcal{E}_{i j}$ are respectively, the production, transfer, redistribution, diffusion and dissipation terms. All the expressions of the terms appearing in Eq. (4) can be identified using a mathematical-physics formalism developed in the spectral space (Chaouat and Schiestel, 2007). The transport equation for the turbulence energy spectrum $E=\varphi_{j j} / 2$ is simply obtained by tensorial contraction of Eq. (4) leading to

$$
\begin{aligned}
& \frac{\partial E(\boldsymbol{X}, \kappa, t)}{\partial t}+\left\langle u_{j}\right\rangle(\boldsymbol{X}) \frac{\partial E_{i j}(\boldsymbol{X}, \kappa, t)}{\partial X_{j}}=\mathcal{P}(\boldsymbol{X}, \kappa, t) \\
& +\mathcal{T}(\boldsymbol{X}, \kappa, t)+\mathcal{J}(\boldsymbol{X}, \kappa, t)-\mathcal{E}(\boldsymbol{X}, \kappa, t)
\end{aligned}
$$

where $\mathcal{P}=\mathcal{P}_{m m} / 2, \mathcal{T}=\mathcal{T}_{m m} / 2, \mathcal{J}=\mathcal{J}_{m m} / 2$ and finally, $\mathcal{E}=\mathcal{E}_{m m} / 2$. Partial integration can be performed on wave number ranges such as $\left[0, \kappa_{c}\right],\left[\kappa_{c}, \kappa_{d}\right]$ and $\left[\kappa_{d}, \infty\left[\right.\right.$ where $\kappa_{c}$ denotes the cutoff wave number given by the grid size $\Delta$ and $\kappa_{d}$ is the dissipative wave number located at the far end of the inertial range of the spectrum assuming that the energy pertaining to higher wave numbers is negligible. Taking into account the significant processes of turbulence acting in each spectral zone of the density spectrum, it is then possible with some calculus to derive the equations associated with the subfilterscale and dissipation rate as demonstrated step by step by Schiestel and Dejoan (2005) as well as Chaouat and Schiestel $(2005,2012)$.

\subsection{Equation set for the turbulent dynamic field}

As a result of modeling (Schiestel and Dejoan, 2005; Chaouat and Schiestel, 2005, 2012), the transport equation for the subfilter stress tensor $\left(\tau_{i j}\right)_{s f s}$ can be written in the simple compact form as (Chaouat and Schiestel, 2005)

$$
\frac{\partial\left(\tau_{i j}\right)_{s f s}}{\partial t}+\frac{\partial}{\partial x_{k}}\left(\bar{u}_{k}\left(\tau_{i j}\right)_{s f s}\right)=\left(P_{i j}\right)_{s f s}+\left(\Pi_{i j}\right)_{s f s}+J_{i j}-\epsilon_{i j}
$$

where the terms appearing in the right-hand side of this equation are identified as subfilter production, redistribution, diffusion and dissipation, respectively. The transport equation for the subfilter turbulent 
energy is obtained as half the trace of Eq. (6)

$$
\frac{\partial k_{s f s}}{\partial t}+\frac{\partial}{\partial x_{k}}\left(\bar{u}_{k} k_{s f s}\right)=P_{s f s}+J-\epsilon
$$

where $P_{s f s}=\left(P_{m m}\right)_{s f s} / 2, J=J_{m m} / 2, \epsilon=\epsilon_{m m} / 2$. The production term $\left(P_{i j}\right)_{s f s}$ accounts for the interaction between the subfilter stresses and the filtered velocity gradients

$$
\left(P_{i j}^{1}\right)_{s f s}=-\left(\tau_{i k}\right)_{s f s} \frac{\partial \bar{u}_{j}}{\partial x_{k}}-\left(\tau_{j k}\right)_{s f s} \frac{\partial \bar{u}_{i}}{\partial x_{k}}
$$

The redistribution term $\left(\Pi_{i j}\right)_{s f s}$ appearing in Eq. (6) is decomposed into a slow part $\left(\Pi_{i j}^{1}\right)_{s f s}$ that characterizes the return to isotropy due to the action of subgrid turbulence on itself

$$
\left(\Pi_{i j}^{1}\right)_{s f s}=-c_{1 s f s} \frac{\epsilon}{k_{s f s}}\left(\left(\tau_{i j}\right)_{s f s}-\frac{1}{3}\left(\tau_{m m}\right)_{s f s} \delta_{i j}\right)
$$

and a rapid part, $\left(\Pi_{i j}^{2}\right)_{s f s}$ that describes the action of the filtered velocity gradients

$$
\left(\Pi_{i j}^{2}\right)_{s f s}=-c_{2}\left(\left(P_{i j}\right)_{s f s}-\frac{1}{3}\left(P_{m m}\right)_{s f s} \delta_{i j}\right)
$$

where $c_{1 s f s}=c_{1} \alpha(\eta)$ is an increasing function of the parameter $\eta$ to strengthen the return to isotropy for large wave numbers, $c_{1}$ and $c_{2}$ are constant coefficients. The closure of the rapid term in the pressurestrain correlations is inspired from the stress transport model developed by Launder et al. (1975) referring to the so-called "isotropization of production "which is a simplified form of the "quasi isotropic "version proposed in the same paper. A similar modeling was obtained by Jeandel et al. [30] and Cambon et al. (1981) for spectral closures. These closures are depending on a single numerical parameter only. Reflection of the difficulty of the task, many highly refined proposals for modeling this term have been proposed up to the intractable (see for instance Schiestel, 2008). Moreover, a recent study conducted by Mishra et al. (2017) contributes to validate a closure approximation with a single numerical parameter similar to the previously cited models. In the case of subfilter quantities, considered here, the influence of the cutoff on this linear term is implicitly contained in the $\left(P_{i j}\right)_{s f s}$ term. The diffusion terms $J_{i j}$ is modeled assuming the well-known gradient law

$$
J_{i j}=\frac{\partial}{\partial x_{m}}\left(\nu \frac{\partial\left(\tau_{i j}\right)_{s f s}}{\partial x_{m}}+c_{k} \frac{k_{s f s}}{\epsilon}\left(\tau_{m l}\right)_{s f s} \frac{\partial\left(\tau_{i j}\right)_{s f s}}{\partial x_{l}}\right)
$$


where $c_{k}$ is a numerical coefficient. The subfilter tensorial dissipation rate $\epsilon_{i j}$ is approached by $2 / 3 \epsilon \delta_{i j}$ at high Reynolds number. Its transport equation reads (Schiestel and Dejoan, 2005; Chaouat and Schiestel, 2005, 2009, 2012)

$$
\frac{\partial \epsilon}{\partial t}+\frac{\partial}{\partial x_{k}}\left(\bar{u}_{k} \epsilon\right)=c_{\epsilon_{1 s f s}} \frac{\epsilon}{k_{s f s}} P_{s f s}-c_{\epsilon_{2 s f s}} \frac{\epsilon^{2}}{k_{s f s}}+J_{\epsilon}
$$

The coefficient appearing in the destruction term of Eq. (12) is then given by (Chaouat and Schiestel, 2009; Chaouat, 2017)

$$
c_{\epsilon_{2 s f s}}=c_{\epsilon_{1}}+\frac{\Delta c_{\epsilon}}{\left[1+\beta \vartheta_{c}^{\alpha}\right]^{\gamma}}
$$

where $\alpha \gamma=2 / 3$ and $\beta=\left[2 /\left(3 C_{K}\right)\right]^{\gamma}, C_{K}$ is the Kolmogorov constant close to $1.5, \vartheta_{c}=\kappa_{c} L$ where $L$ denotes the turbulence length-scale while $\kappa_{c}$ is the cutoff wave number computed using the grid size of the mesh $\Delta$ as $\kappa_{c}=\pi / \Delta, \Delta c_{\epsilon}=c_{\epsilon_{2}}-c_{\epsilon_{1}}, c_{\epsilon_{1}}$ and $c_{\epsilon_{2}}$ are the coefficients used in RANS with the values $c_{\epsilon_{1}}=1.50$ and $c_{\epsilon_{2}}=1.90$, respectively. This function introduced in Eq. (13) allows to sensitize the model to the filter width. Then, the coefficient $c_{\epsilon_{2 s f s}}$ can be considered as a dynamical parameter which draws the spectral distribution towards the prescribed equilibrium distribution. In other words, this term acts like a relaxation towards the Kolmogorov equilibrium spectrum (Chaouat and Schiestel, 2012). The coefficient $c_{\epsilon_{1 s f s}}$ is the same as the one used in the corresponding RANS dissipation equation $c_{\epsilon_{1 s f s}}=c_{\epsilon_{1}}$. The term $J_{\epsilon}$ denotes the diffusion term that is modeled using a tensorial gradient law.

$$
J_{\epsilon}=\frac{\partial}{\partial x_{j}}\left(\nu \frac{\partial \epsilon}{\partial x_{j}}+c_{\epsilon} \frac{k_{s f s}}{\epsilon}\left(\tau_{j m}\right)_{s f s} \frac{\partial \epsilon}{\partial x_{m}}\right)
$$

where $c_{\epsilon}$ is a numerical coefficient. The subfilter model relying on Eqs. (6) and (12) has been also extended to low Reynolds numbers for approaching walls (see Chaouat and Schiestel, 2013).

\section{Variance of the turbulent passive scalar field in spectral space}

The new PITM development of the dissipation rate equation for the passive scalar variance is founded in spectral space in a way similar to the development of the dissipation rate equation for kinetic energy introduced in earlier works, and for this reason it will be considered first. The transported scalar is 
considered as passive with no direct action on the flow dynamics. It can represent temperature or any other transported contaminant and will be denoted $\theta$. The main quantity representing the properties of turbulent scalar distribution is the variance of the scalar which is the counterpart of the kinetic energy of turbulence. The half variance $k_{\theta}$ is defined as $k_{\theta}(\boldsymbol{X}, t)=\left\langle\theta^{\prime} \theta^{\prime}(\boldsymbol{X}, t)\right\rangle / 2$ where $\theta^{\prime}$ denotes the usual fluctuation appearing in statistical treatment. We consider splitting of the spectrum of the variance of the scalar as shown in Fig. 2. The spectral transport equation of the half scalar variance denoted

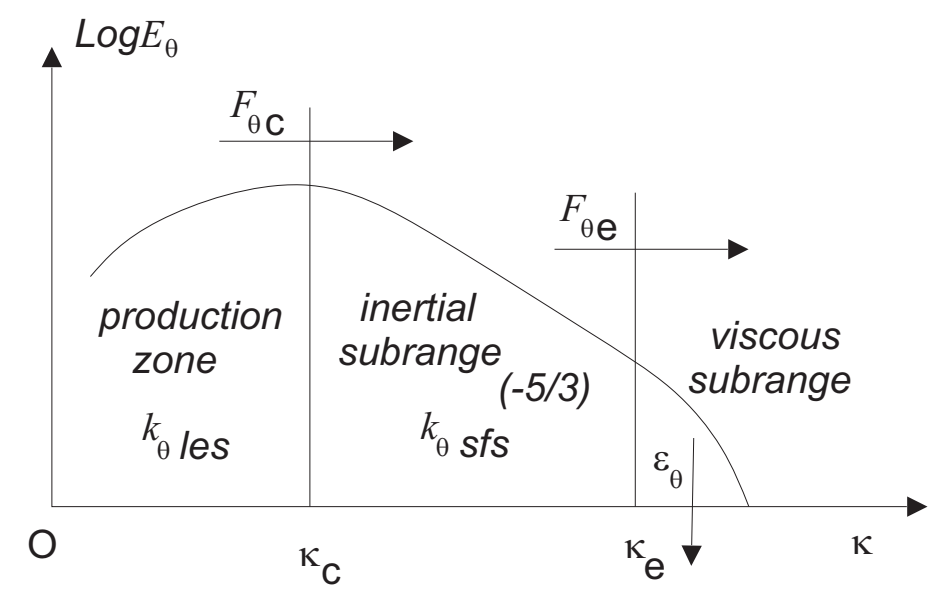

Figure 2: Sketch of spectral splitting of scalar variance

$E_{\theta}(\boldsymbol{X}, \kappa, t)=\left\langle\theta^{\prime} \theta^{\prime}(\boldsymbol{X})\right\rangle^{\Delta}(\kappa, t) / 2$ for the mean kinetic energy spectrum in homogeneous anisotropic turbulence and has been established in A according to previous works (Tennekes and Lumley, 1972; Monin and Yaglom, 1975; Schiestel, 1983a, 1983b; Mathieu et al., 1984; Launder et al., 84b). This equation reads

$$
\begin{aligned}
& \frac{\partial E_{\theta}(\boldsymbol{X}, \kappa, t)}{\partial t}+\left\langle u_{k}\right\rangle(\boldsymbol{X}) \frac{\partial E_{\theta}(\boldsymbol{X}, \kappa, t)}{\partial X_{k}}=\mathcal{P}_{\theta}(\boldsymbol{X}, \kappa, t)+\mathcal{T}_{\theta}(\boldsymbol{X}, \kappa, t) \\
& +\mathcal{J}_{\theta}(\boldsymbol{X}, \kappa, t)-\mathcal{E}_{\theta}(\boldsymbol{X}, \kappa, t)
\end{aligned}
$$

where in the right hand side of this equation, $\mathcal{P}_{\theta}$ is the production of variance of the scalar by mean gradients of the scalar, $\mathcal{T}_{\theta}$ is the spectral transfer entrained by the eddying motions in the inertial cascade, $\mathcal{J}_{\theta}$ is the diffusion term and $\mathcal{E}_{\theta}$ denotes the dissipation term of the scalar variance. Without loosing generality, but for sake of simplification, we restrict provisionally the analysis to homogeneous 
flows so that the diffusion term vanishes and the variable $\boldsymbol{X}$ is omitted in the following. However, for practical non-homogeneous turbulent flows applications, the tangent homogeneous space approximation will be used. The production term $\mathcal{P}_{\theta}$ detailed in equation (74) of A involves the turbulent scalar fluxes $\varphi_{i \theta}(\boldsymbol{X}, \kappa, t)$ which have to be modeled carefully because they control the transport properties of turbulence. A physical approximation inspired from the GGDH hypothesis of Launder (1988) leads to

$$
\varphi_{i \theta}(\boldsymbol{X}, \kappa, t)=-\varphi_{i j}(\boldsymbol{X}, \kappa, t) \tau(\boldsymbol{X}, t) \frac{\partial\langle\theta\rangle}{\partial x_{j}}
$$

where $\tau(\boldsymbol{X}, t)$ is a characteristic time scale of turbulence. The hypothesis will be detailed further in the next section devoted to the physical space. The PITM method then uses a spectral decomposition where the wave number splitting can be viewed as a spectral filter (Yoshizawa, 1982). Like in multiple-scale models (Schiestel, 1988), the spectral equation (15) can be partially integrated over the wave number range such as $\left[0, \kappa_{c}\right],\left[\kappa_{c}, \kappa_{e}\right]$ and $\left[\kappa_{e}, \infty\left[\right.\right.$ where $\kappa_{e}$ denotes a wave number that is larger than $\kappa_{c}$ but it can be different from $\kappa_{d}$. As a result, one can obtain the following approximate equations

$$
\begin{gathered}
\frac{\partial k_{\theta l e s}}{\partial t}=P_{\theta\left[0, \kappa_{c}\right]}-F_{\theta}\left(\kappa_{c}, t\right) \\
\frac{\partial k_{\theta s f s}}{\partial t}=P_{\theta\left[\kappa_{c}, \kappa_{e}\right]}-F_{\theta}\left(\kappa_{e}, t\right)+F_{\theta}\left(\kappa_{c}, t\right) \\
0=F_{\theta}\left(\kappa_{e}, t\right)-\epsilon_{\theta\left[\kappa_{e}, \infty[\right.}
\end{gathered}
$$

where

$$
\begin{gathered}
k_{\theta s f s}=\int_{\kappa_{c}}^{\kappa_{e}} E_{\theta}(\kappa, t) d \kappa, \quad k_{\theta l e s}=\int_{0}^{\kappa_{c}} E_{\theta}(\kappa, t) d \kappa, \quad k_{\theta}=k_{\theta l e s}+k_{\theta s f s} \\
F_{\theta}\left(\kappa_{c}, t\right)=\mathcal{F}_{\theta}\left(\kappa_{c}, t\right)-E_{\theta}\left(\kappa_{c}, t\right) \frac{\partial \kappa_{c}}{\partial t} \\
F_{\theta}\left(\kappa_{e}, t\right)=\mathcal{F}_{\theta}\left(\kappa_{e}, t\right)-E_{\theta}\left(\kappa_{e}, t\right) \frac{\partial \kappa_{e}}{\partial t}
\end{gathered}
$$

and

$$
\epsilon_{\theta\left[\kappa_{e}, \infty\right]}=\int_{\kappa_{e}}^{\infty} \mathcal{E}_{\theta}(\kappa, t) d \kappa
$$

where $\epsilon_{\theta\left[\kappa_{e}, \infty\right]} \approx \epsilon_{\theta}$. So that Eq. (18) can be rewritten as

$$
\frac{\partial k_{\theta s f s}}{\partial t}=P_{\theta\left[\kappa_{c}, \kappa_{e}\right]}+F_{\theta}\left(\kappa_{c}, t\right)-\epsilon_{\theta}
$$


Equations (21) and (22) result from the derivative of the integral with variable bounds. The total variance transfer $F_{\theta}\left(\kappa_{e}, t\right)$ through the variable cutoff $\kappa_{e}$ is equal to the sum of the local spectral flux $\mathcal{F}_{\theta}\left(\kappa_{e}, t\right)$ and the transfer due to the variation in the splitting wavenumber. At the wavenumber $\kappa_{e}$, all the preceding hypotheses imply $F_{\theta}\left(\kappa_{e}\right) \approx \epsilon_{\theta}$, the turbulence Reynolds number being supposed to be large. For the scalar variance, the order of magnitude of the leading terms in Eq. (24) is $\mathcal{O}\left(\theta^{2} u / l_{\theta}\right)$ where $l_{\theta}$ is the turbulence macroscale involved in the scalar physical processes. Consequently, $\kappa_{e}-\kappa_{c}=\mathcal{O}\left(1 / l_{\theta}\right)=\mathcal{O}\left(\epsilon_{\theta} / \theta^{2} u\right)$ leading to the equation

$$
\kappa_{e}-\kappa_{c}=\zeta_{\theta} \frac{\epsilon_{\theta}}{k_{\theta s f s} k_{s f s}^{1 / 2}}
$$

where $\zeta_{\theta}$ is an adjustable coefficient chosen such that the spectral contribution of the variance beyond $\kappa_{e}$ is negligible. Equation (25) is the scalar counterpart of the dynamical equation already developed in the dynamical model (Schiestel and Dejoan, 2005; Chaouat and Schiestel, 2005). Combining these equations together yields

$$
\begin{aligned}
& \frac{\partial \epsilon_{\theta}}{\partial t}=\frac{\epsilon_{\theta}}{k_{\theta s f s}} \frac{\partial k_{\theta s f s}}{\partial t}+\frac{\epsilon_{\theta}}{2 k_{s f s}} \frac{\partial k_{s f s}}{\partial t}+ \\
& \frac{\epsilon_{\theta}}{\kappa_{e}-\kappa_{c}}\left[\frac{\mathcal{F}_{\theta}\left(\kappa_{e}, t\right)-F_{\theta}\left(\kappa_{e}, t\right)}{E_{\theta}\left(\kappa_{e}, t\right)}-\frac{\mathcal{F}_{\theta}\left(\kappa_{c}, t\right)-F_{\theta}\left(\kappa_{c}, t\right)}{E_{\theta}\left(\kappa_{c}, t\right)}\right]
\end{aligned}
$$

Using the transport equations for $k_{s f s}$ and $\epsilon$ recalled in section 2.4 and Eq. (24), one can obtain the resulting equation for the dissipation-rate $\epsilon_{\theta}$ written in a more compact form as

$$
\frac{\partial \epsilon_{\theta}}{\partial t}=c_{\epsilon_{\theta \theta_{1} s f s}} P_{\theta s f s} \frac{\epsilon_{\theta}}{k_{\theta s f s}}+c_{\epsilon_{\theta k_{1} s f s}} P_{s f s} \frac{\epsilon_{\theta}}{k_{s f s}}-c_{\epsilon_{\theta k_{2} s f s}} \frac{\epsilon_{\theta} \epsilon}{k_{s f s}}-c_{\epsilon_{\theta \theta_{2} s f s}} \frac{\epsilon_{\theta}^{2}}{k_{\theta s f s}}
$$

where

$$
\begin{gathered}
P_{\theta s f s}=P_{\theta\left[\kappa_{c}, \kappa_{e}\right]}+F_{\theta}\left(\kappa_{c}\right) \\
c_{\epsilon_{\theta \theta_{1} s f s}}=1 \quad, \quad c_{\epsilon_{\theta k_{1} s f s}}=\frac{1}{2} \quad, \quad c_{\epsilon_{\theta k_{2} s f s}}=\frac{1}{2}
\end{gathered}
$$

and

$$
\begin{aligned}
& c_{\epsilon_{\theta \theta_{2} s f s}}=1-\frac{k_{\theta s f s}}{\left(\kappa_{e}-\kappa_{c}\right) E_{\theta}\left(\kappa_{e}\right)} \\
& {\left[\left(\frac{\mathcal{F}_{\theta}\left(\kappa_{e}\right)}{\epsilon_{\theta}}-\frac{F_{\theta}\left(\kappa_{e}\right)}{\epsilon_{\theta}}\right)-\frac{E_{\theta}\left(\kappa_{e}\right)}{E_{\theta}\left(\kappa_{c}\right)} \frac{F_{\theta}\left(\kappa_{c}\right)}{\epsilon_{\theta}}\left(\frac{\mathcal{F}_{\theta}\left(\kappa_{c}\right)}{F_{\theta}\left(\kappa_{c}\right)}-1\right)\right]}
\end{aligned}
$$


Setting $\kappa_{c} \ll \kappa_{e}, E\left(\kappa_{d}\right) \ll E\left(\kappa_{c}\right)$, and $E_{\theta}\left(\kappa_{e}\right) \ll E_{\theta}\left(\kappa_{c}\right)$, and also considering that $F_{\theta}\left(\kappa_{e}\right)=\epsilon_{\theta}$ as indicated by Eq. (19), Eq. (30) reduces then to

$$
c_{\epsilon_{\theta \theta_{2} s f s}}=1-\frac{k_{\theta s f s}}{\kappa_{e} E_{\theta}\left(\kappa_{e}\right)}\left(\frac{\mathcal{F}_{\theta}\left(\kappa_{e}\right)}{\epsilon_{\theta}}-1\right)
$$

When $\kappa_{c}$ goes to zero, that is to say when the filter width in physical space goes to infinity in an homogeneous turbulence field (or locally homogeneous), one recovers the equation used in statistical RANS closure. Hence, the equation can be written as

$$
\frac{\partial \epsilon_{\theta}}{\partial t}=c_{\epsilon_{\theta \theta_{1}}} P_{\theta} \frac{\epsilon_{\theta}}{k_{\theta}}+c_{\epsilon_{\theta k_{1}}} P \frac{\epsilon_{\theta}}{k}-c_{\epsilon_{\theta k_{2}}} \frac{\epsilon_{\theta} \epsilon}{k}-c_{\epsilon_{\theta \theta_{2}}} \frac{\epsilon_{\theta}^{2}}{k_{\theta}}
$$

where

$$
c_{\epsilon_{\theta \theta_{1}}}=1 \quad, \quad c_{\epsilon_{\theta k_{1}}}=\frac{1}{2} \quad, \quad c_{\epsilon_{\theta k_{2}}}=\frac{1}{2}
$$

and

$$
c_{\epsilon_{\theta \theta_{2}}}=1-\frac{k_{\theta}}{\kappa_{e} E_{\theta}\left(\kappa_{e}\right)}\left(\frac{\mathcal{F}_{\theta}\left(\kappa_{e}\right)}{\epsilon_{\theta}}-1\right)
$$

Several modeling papers from the 1970's have proposed modeled forms of the dissipation-rate of the scalar. One of the most complete forms can be found in the work of Yoshizawa (1988) and also several authors such as Nagano and Kim (1988) as well as Shikazono and Kasagi (1996). In the earlier formulations of Eq. (32), it happened that some terms were obviously missing. But the form of Eq. (32) obtained in the present case by a theoretical formalism seems indeed to be one of the most general considering the different source terms involved in this equation (Newmann et al., 1981; Jones and Musonge, 1988). Physically, the value of $\epsilon_{\theta}$ which can be viewed as the flux of variance in the inertial cascade must remain the same whatever the location of the spectral splitting. Comparing Eq. (31) with Eq. (34) leads to

$$
c_{\epsilon_{\theta \theta_{2} s f s}}=c_{\epsilon_{\theta \theta_{1}}}+\frac{k_{\theta s f s}}{k_{\theta}}\left(c_{\epsilon_{\theta \theta_{2}}}-c_{\epsilon_{\theta \theta_{1}}}\right)
$$

where the numerical value $c_{\epsilon_{\theta \theta_{1}}}$ supposed to take on the value unity can be slightly changed in practice. As a result of interest, one can remark that Eqs. (27) and (32) have exactly the same general form as the usual modeled equation of the dissipation of variance found in the open literature and especially in Refs. (Yoshizawa, 1988; Nagano and Kim, 1988) but also in Refs. (Schiestel, 2007; Mathieu et al., 1984b; Launder et al., 1984b; Ruffin et al., 1994; Gharbi et al., 1996). The development of the PITM 
method leads to find that it is the $c_{\epsilon_{\theta_{2}} s f s}$ coefficient that controls the partitioning of scalar variance like the $c_{\epsilon_{2 s f s}}$ coefficient in the epsilon equation was controlling the kinetic energy. It is not an initial choice but the result of the development of the method.

\section{Parametrization of the variable coefficients in the passive scalar dissipation equations}

\subsection{Molecular Prandtl numbers near unity}

The ratio $k_{\theta s f s} / k_{\theta}$ appearing in Eq. (35) must be calibrated as a function of the location of the cutoff wavenumber. As indicated in Refs. (Corrsin, 1964; Tennekes and Lumley, 1972) for Prandtl number near to unity, the spectrum of the scalar in the equilibrium range can be approximated by

$$
E_{\theta}(\kappa)=C_{\theta} \epsilon_{\theta} \epsilon^{-1 / 3} \kappa^{-5 / 3}
$$

where $C_{\theta}$ is a constant coefficient close to 0.5 . The value of this numerical constant remains however approximate. Even to these present days, it is still not accurately well calibrated and is subject to discussion in the literature as pointed out by Favre et al. (1976). The spectrum of the scalar $\theta$ given by Eq. (36) can be extended in the whole range domain of the wavenumbers using the very likely hypothesis

$$
E_{\theta}(\kappa)=\frac{C_{\theta} \epsilon_{\theta}}{C_{K} \epsilon} E(\kappa)
$$

where $E(\kappa)$ is the energy density spectrum of turbulence. Equation (37) must verify the limiting condition $\lim _{\kappa \rightarrow \infty} E_{\theta}(\kappa)=C_{\theta} \epsilon_{\theta} \epsilon^{-1 / 3} \kappa^{-5 / 3}$. The analytical integration made in B.1 yields the practical result

$$
c_{\epsilon_{\theta \theta_{2} s f s}}=c_{\epsilon_{\theta \theta_{1}}}+\frac{\Delta c_{\epsilon_{\theta \theta}}}{\left[1+\beta \vartheta_{c}^{\alpha}\right]^{\gamma}}
$$

where $\Delta c_{\epsilon_{\theta \theta}}=c_{\epsilon_{\theta \theta_{2}}}-c_{\epsilon_{\theta \theta_{1}}}$. As expected, the result is analogous to the formula previously obtained for the dynamical equations for kinetic energy. We mention also that for $P_{r}=1$, Eq. (37) reflects an analogy between the velocities and scalar fields corresponding to the well known Reynolds analogy. 


\subsection{Small molecular Prandtl numbers}

This situation corresponds to the case of liquid metals that can have specific industrial applications. In this case, the inertial subrange of the variance spectrum is shorter and terminated by a steep inertial diffusive subrange. In this subrange, the spectral flux of variance of the scalar decreases drastically because of the dissipation of variance due to high molecular diffusivity. As indicated in Refs. (Batchelor, 1959; Tennekes and Lumley, 1972) the spectrum of the scalar variance can be represented by the function

$$
E_{\theta}(\kappa)=C_{\theta} \epsilon_{\theta} \epsilon^{-1 / 3} \kappa^{-5 / 3} \exp \left[-\frac{3}{2} C_{\theta}\left(\kappa \eta_{\theta}\right)^{4 / 3}\right]
$$

with the scalar microscale defined by $\eta_{\theta}=\left(\sigma^{3} / \epsilon\right)^{1 / 4}$ where $C_{\theta}=1.5$ is a constant coefficient such that $C_{\theta}=k_{\theta} C_{K} \epsilon /\left(k \epsilon_{\theta}\right)$ as demonstrated in Eq. (81). Introducing the Kolmorogov scale $\eta_{K}=\left(\nu^{3} / \epsilon\right)^{1 / 4}$ and the Prandtl number $P_{r}=\nu / \sigma$ indicated in Refs. (Batchelor, 1959; Tennekes and Lumley, 1972; Hinze, 1975; Monin and Yaglom, 1975), the scalar microscale can be computed by $\eta_{\theta}=\eta_{K} / P_{r}^{3 / 4}$. The spectrum shape associated with Eq. (39) is shown in Fig. 3. Dealing with an exponential factor

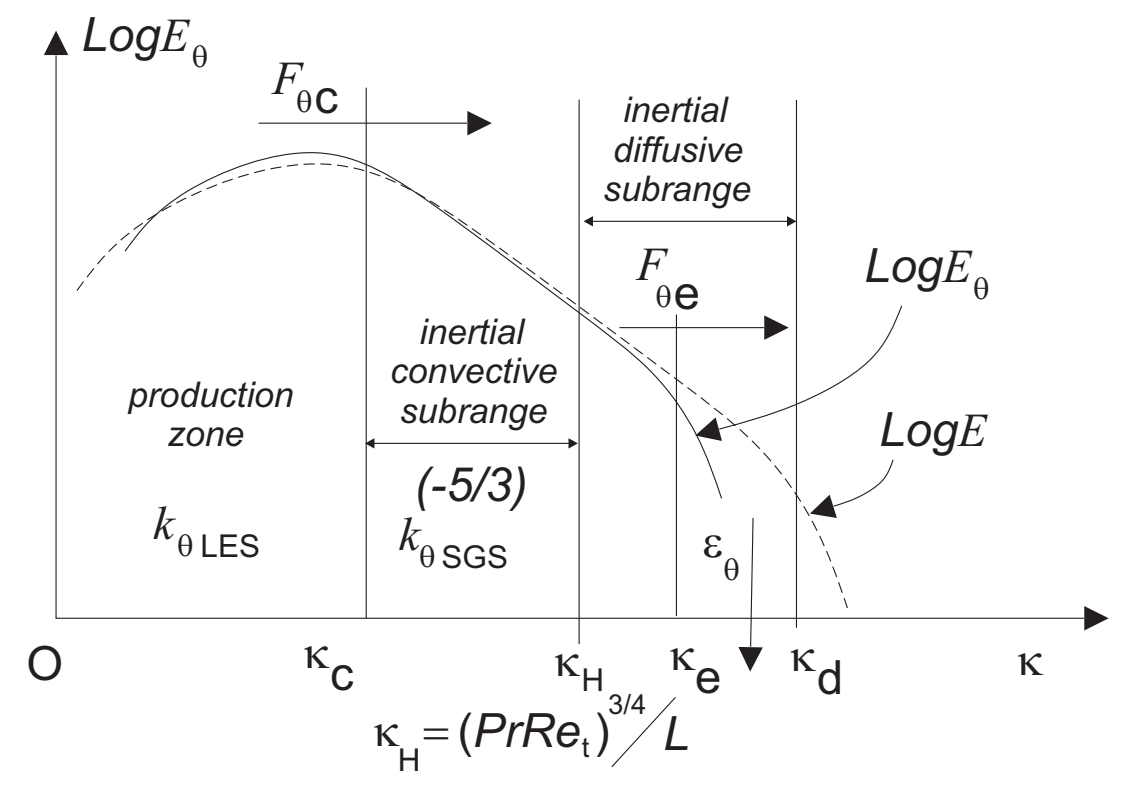

Figure 3: Spectral zones for small molecular Prandtl numbers where $\kappa_{H}=\left(\epsilon / \sigma^{3}\right)^{1 / 4}$ and $L=k^{3 / 2} / \epsilon$. 
results in cumbersome formulas in the model equations. So, in practice a simpler approach is retained. Equation (39) is then replaced by

$$
E_{\theta}(\kappa)=C_{\theta} \epsilon_{\theta} \epsilon^{-1 / 3} \kappa^{-5 / 3} H\left(\kappa_{H}-\kappa\right)
$$

where $\kappa_{H}=1 / \eta_{\theta}$, and $H$ is the Heaviside function implying that $E_{\theta}(\kappa)=0$ for $\kappa \geq \kappa_{H}$. The spectral vanishing value of wavenumber is then obtained for $\kappa \eta_{\theta}=1$. So that the dimensionless variable $\vartheta$ is dropping for $\vartheta_{H}=\left(P_{r} R e_{t}\right)^{3 / 4}$ where $R e_{t}=k^{2} / \nu \epsilon$ denotes the turbulent Reynolds number. This dropping value can be expressed equivalently as $\vartheta_{H}=k^{3 / 2} /\left(\eta_{\theta} \epsilon\right)=\left(\sigma k^{2} / \epsilon\right)^{3 / 4}$. Physically, the dimensionless group $P_{r} R e_{t}$ is interpreted like the turbulent Peclet number denoted $P e_{t}=P_{r} R e_{t}$. Obviously, as seen in Fig. 4, the value of $\kappa_{e}$ can be larger or smaller than $\eta_{\theta}^{-1}$. The spectrum (40) valid in the inertial law can be extended in the whole wavenumber range by writing

$$
E_{\theta}(\kappa)=\frac{C_{\theta} \epsilon_{\theta}}{C_{K} \epsilon} E(\kappa) H\left(\kappa_{H}-\kappa\right)
$$

where $E(\kappa)$ is the spectrum valid in the whole wave number range given by Eq. (83). The exact final expression of the coefficient $c_{\epsilon_{\theta \theta_{2}} s f s}$ is developed in B.2 and reads

$$
c_{\epsilon_{\theta \theta_{2} s f s}}= \begin{cases}c_{\epsilon_{\theta \theta_{1}}}+\Delta c_{\epsilon_{\theta \theta}} \frac{\left[1+\beta \vartheta_{c}^{\alpha}\right]^{-\gamma}-\left[1+\beta P e_{t}^{3 \alpha / 4}\right]^{-\gamma}}{1-\left[1+\beta P e_{t}^{3 \alpha / 4}\right]^{-\gamma}} & \left(\vartheta_{c}<\vartheta_{H}\right) \\ c_{\epsilon_{\theta \theta_{1}}} & \left(\vartheta_{c}>\vartheta_{H}\right)\end{cases}
$$

\subsection{Large molecular Prandtl numbers}

This situation corresponds to the case of poorly conducting fluids or high viscous fluids like most of oils. In this case, the inertial subrange is followed by a viscous-convective subrange with a negative slope of minus unity and finally a viscous-diffusive subrange in which the spectrum undergoes strong decay as indicated in Refs. (Batchelor, 1959; Tennekes and Lumley, 1972). In the viscous-convective subrange, the scalar fluctuations are broken and decreased by the strain-rate field but the scalar diffusivity is not yet active. The shape of the spectrum has been studied by Batchelor (1959). For the wave number $\kappa \geq 1 / \eta_{K}$, it can be shown that the viscous convective subrange of the spectrum is of the form

$$
E_{\theta}(\kappa)=c_{\theta} \epsilon_{\theta}\left(\frac{\nu}{\epsilon}\right)^{1 / 2} \kappa^{-1}
$$


where $c_{\theta}$ is a constant coefficient. The viscous convective subrange is followed by the viscous-diffusive subrange which is characterized by the role of scalar diffusivity acting on very small scales. In this region, the spectrum takes on the form

$$
E_{\theta}(\kappa)=c_{\theta} \epsilon_{\theta}\left(\frac{\nu}{\epsilon}\right)^{1 / 2} \kappa^{-1} \exp \left[-c_{\theta}\left(\kappa \eta_{\theta}^{*}\right)^{2}\right]
$$

where $\eta_{\theta}^{*}=\eta_{K}(\sigma / \nu)^{1 / 2}=\eta_{K} / \sqrt{P_{r}}$ is the smallest scale of the viscous-diffusive subrange and $c_{\theta}$ is a constant coefficient. The corresponding wave numbers are then computed as $\kappa_{K}=1 / \eta_{K}$ and $\kappa_{S}=1 / \eta_{\theta}^{*}$. The junctions between the different subranges occur for $\kappa=\kappa_{K}$ and $\kappa=\kappa_{S}$ respectively preserving continuity. In particular, for $\kappa=\kappa_{K}$, the spectrum $E_{\theta}\left(\kappa_{K}\right)$ given by Eq. (36) of the scalar in the equilibrium range with a slope $\kappa^{-5 / 3}$ is equal to the spectrum $E_{\theta}\left(\kappa_{K}\right)$ given by Eq. (43) of the viscousconvective subrange with a slope $\kappa^{-1}$ so that it is found that $c_{\theta}=C_{\theta} \approx 1.5$. The dimensionless wave numbers $\vartheta=\kappa L$ associated with the Kolmogorov scale $\eta_{K}$ and the smallest scale $\eta_{\theta}^{*}$ are $\vartheta_{K}=R e_{t}^{3 / 4}$ and $\vartheta_{S}=P_{r}^{1 / 2} R e_{t}^{3 / 4}$, respectively. In practice, a simple approach is retained so that the spectrum given

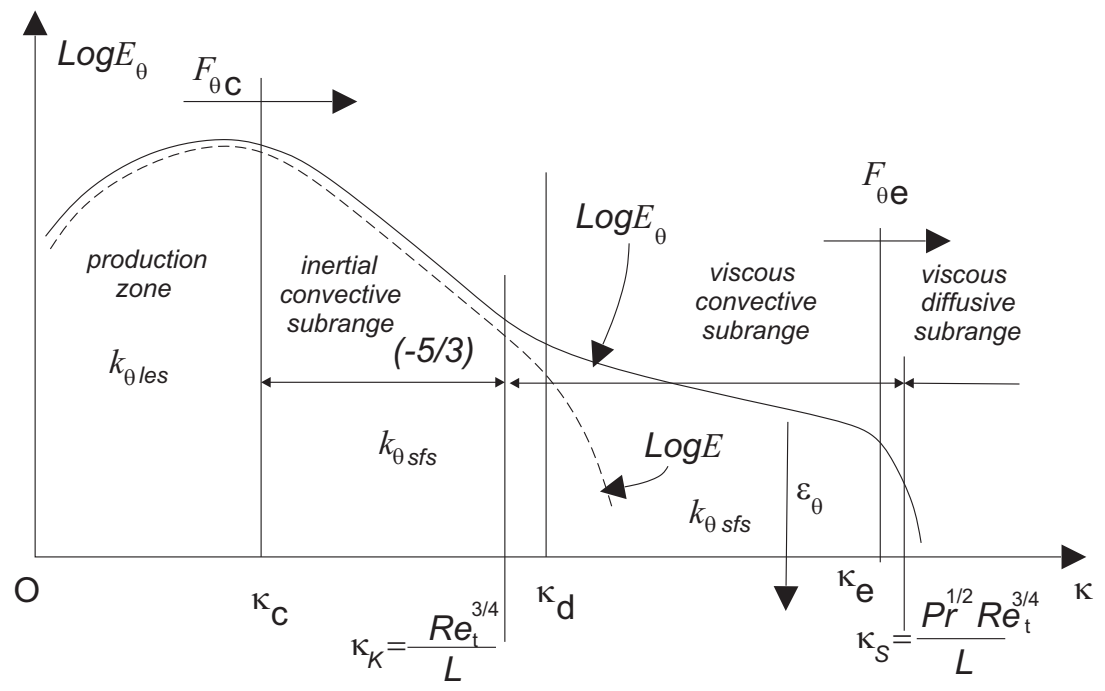

Figure 4: Spectral zones for large molecular Prandtl numbers where $\kappa_{K}=1 / \eta_{K}$ and $\kappa_{S}=P_{r}^{1 / 2} / \eta_{K}$ with $\eta_{K}=\left(\nu^{3} / \epsilon\right)^{1 / 4}$ and $L=k^{3 / 2} / \epsilon$.

by Eq. (44) is replaced by a simple form as

$$
E_{\theta}(\kappa)=c_{\theta} \epsilon_{\theta}\left(\frac{\nu}{\epsilon}\right)^{1 / 2} \kappa^{-1} H\left(\kappa_{S}-\kappa\right)
$$


implying that $E(\kappa)=0$ for $\kappa \geq \kappa_{S}$. In the wave number range $\left[0, \kappa_{K}\right]$, the spectrum still evolves according to Eq. (83). The wave number range $\left[0, \kappa_{S}\right]$ is then decomposed into two wave number ranges introducing the cutoff wave number $\kappa_{c}$ where $\kappa_{c}<\kappa_{S}$ or $\kappa_{c}>\kappa_{S}$. In the first wave number range $\left[0, \kappa_{K}\right]$, the spectrum $E_{\theta_{1}}(\kappa)$ is defined as

$$
E_{\theta_{1}}(\kappa)=\xi \frac{k_{\theta}}{k} E(\kappa)
$$

where $E(\kappa)$ is given by Eq. (83) whereas in the second domain $\left[\kappa_{K}, \infty\left[\right.\right.$, the spectrum $E_{\theta_{2}}(\kappa)$ is deduced from Eq. (45)

$$
E_{\theta_{2}}(\kappa)=\xi c_{\theta} \epsilon_{\theta}\left(\frac{\nu}{\epsilon}\right)^{1 / 2} \kappa^{-1} H\left(\kappa_{S}-\kappa\right)
$$

where $\xi$ is a coefficient of normalization. After an analytical integration made in B.3, we obtain the exact expression for the $c_{\epsilon_{\theta \theta_{2}} s f s}$ coefficient

$$
c_{\epsilon_{\theta \theta_{2} s f s}}=\left\{\begin{array}{lc}
c_{\epsilon_{\theta \theta_{1}}}+\Delta c_{\epsilon_{\theta \theta}} \frac{\left[1+\beta \vartheta_{c}^{\alpha}\right]^{-\gamma}-\left[1+\beta R e_{t}^{3 \alpha / 4}\right]^{-\gamma}+c_{\theta} \frac{\epsilon_{\theta}}{k_{\theta}}\left(\frac{\nu}{\epsilon}\right)^{1 / 2} \ln P_{r}^{1 / 2}}{1-\left[1+\beta R e_{t}^{3 \alpha / 4}\right]^{-\gamma}+c_{\theta} \frac{\epsilon_{\theta}}{k_{\theta}}\left(\frac{\nu}{\epsilon}\right)^{1 / 2} \ln P_{r}^{1 / 2}}\left(\vartheta_{C}<\vartheta_{K}\right) \\
c_{\epsilon_{\theta \theta_{1}}+\Delta c_{\epsilon_{\theta \theta}} \frac{\epsilon_{\theta}}{k_{\theta}}\left(\frac{\nu}{\epsilon}\right)^{1 / 2} \ln \frac{\vartheta_{S}}{\vartheta_{c}}} \quad\left(\vartheta_{K}<\vartheta_{C}<\vartheta_{S}\right) \\
c_{\epsilon_{\theta \theta_{1}}} \quad\left(\vartheta_{S}<\vartheta_{C}\right)
\end{array}\right.
$$

Introducing the dimensionless ratio $\mathcal{R}$ that characterizes the turbulence scale of the passive scalar field compared to the dynamic scales (Kenjeres et al., 2005, 2015) $\mathcal{R}=\left(k_{\theta s f_{s}} \epsilon\right) /\left(k_{s f_{s}} \epsilon_{\theta}\right)$ then, the factor $\zeta=\left(\varepsilon_{\theta} / k_{\theta}\right)(\nu / \varepsilon)^{1 / 2}$ involved in equation (96) can be approximated in a simple way using the relations $\left(\varepsilon_{\theta} / k_{\theta}\right)=\varepsilon /(k \mathcal{R})$ and $(\nu / \varepsilon)^{1 / 2}=k /\left(\varepsilon R e_{t}^{1 / 2}\right)$, thus implying

$$
\frac{\varepsilon_{\theta}}{k_{\theta}}\left(\frac{\nu}{\varepsilon}\right)^{1 / 2}=\frac{1}{\mathcal{R}} R e_{t}^{-1 / 2}
$$

At a first sight, it appears that these analytical coefficients seem fairly complicated. But in fact, they can be easily implemented in CFD codes, because the extra computing time is entirely negligible. As emphasized above, this is the price to pay for dealing with an extended Prandtl number range. 


\section{$5 \quad$ Final equation set for passive scalar turbulence}

\subsection{Turbulent passive scalar field equations in physical space}

The transport equation of the subfilter scale scalar variance including additional turbulent diffusion terms in non-homogeneous turbulence analogous to Eq. (24) reads

$$
\frac{\partial k_{\theta s f s}}{\partial t}+\frac{\partial}{\partial x_{k}}\left(\bar{u}_{k} k_{\theta s f s}\right)=P_{\theta s f s}+J_{\theta}-\epsilon_{\theta}
$$

The production term $P_{\theta s f s}$ is given by

$$
P_{\theta s f s}=-\left(\tau_{j \theta}\right)_{s f s} \frac{\partial \bar{\theta}}{\partial x_{j}}
$$

where $\left(\tau_{i \theta}\right)_{s f s}$ represents the turbulent scalar flux vector which is of primary importance for the determination of turbulent transfer properties. In a second order closure framework, it is advisable to solve the transport equation for $\left(\tau_{i \theta}\right)_{s f s}$ but all the problems mentioned previously for the linear part of the pressure-strain correlation are still posed here for the pressure-scalar gradient correlation term $\left(\Pi_{i \theta}\right)_{s f s}$ (Launder et al., 1984, Schiestel, 2007; Gatski, 2009). However, in the present case, as mentioned above, due focus is given to the variance of scalar fluctuation and it is assumed in a preliminary step a generalized gradient diffusion hypothesis (GGDH) first introduced by Daly and Harlow (1970) and often applied in practice by many authors such as Launder (1988), Suga (2004), Kenjeres and Hanjalic (2006), Kenjeres et al. (2015)

$$
\left(\tau_{i \theta}\right)_{s f s}=-c_{\tau_{\theta}}\left(\tau_{i m}\right)_{s f s} \frac{k_{s f s}}{\epsilon} \frac{\partial \bar{\theta}}{\partial x_{m}}
$$

where $c_{\tau_{\theta}}$ is a numerical coefficient. This modeling is in the present case here also strengthened by the fact that the turbulent stress tensor $\tau_{i j}$ appearing in Eq. (52) is computed by a second-moment closure (SMC) which allows to accurately reproduce the turbulence anisotropy and not by simple eddy viscosity models. So, the tensorial diffusivity benefits obviously from the second order closure in turbulent stresses. This is an important point because the turbulent scalar fluxes stand as input in the scalar variance equation and also its dissipation rate. Comparisons of turbulent scalar fluxes to known DNS calculations are given in the following in order to ensure that proper scalar flux predictions are obtained using Eq. (52). As the turbulent stresses are computed in the framework of second moment closure, 
the diffusion term $J_{\theta}$ is modeled also using a well-known tensorial gradient law hypothesis

$$
J_{\theta}=\frac{\partial}{\partial x_{m}}\left(\sigma \frac{\partial k_{\theta s f s}}{\partial x_{m}}+c_{k_{\theta}}\left(\tau_{m j}\right)_{s f s} \frac{k_{s f s}}{\epsilon} \frac{\partial k_{\theta s f s}}{\partial x_{j}}\right)
$$

where $c_{k_{\theta}}$ is a constant coefficient. The final equation of the scalar variance dissipation-rate $\epsilon_{\theta}$ is deduced from Eq. (27) including the additional diffusion term as follows

$$
\frac{\partial \epsilon_{\theta}}{\partial t}=c_{\epsilon_{\theta \theta_{1} s f s}} P_{\theta s f s} \frac{\epsilon_{\theta}}{k_{\theta s f s}}+c_{\epsilon_{\theta k_{1} s f s}} P_{s f s} \frac{\epsilon_{\theta}}{k_{s f s}}-c_{\epsilon_{\theta k_{2} s f s}} \frac{\epsilon_{\theta} \epsilon}{k_{s f s}}-c_{\epsilon_{\theta \theta_{2} s f s}} \frac{\epsilon_{\theta}^{2}}{k_{\theta s f s}}+J_{\epsilon_{\theta}}
$$

where the diffusion term $J_{\epsilon_{\theta}}$ is modeled assuming the tensorial gradient law hypothesis

$$
J_{\epsilon_{\theta}}=\frac{\partial}{\partial x_{m}}\left(\sigma \frac{\partial \epsilon_{\theta}}{\partial x_{m}}+c_{\epsilon_{\theta}}\left(\tau_{m j}\right)_{s f s} \frac{k_{s f s}}{\epsilon} \frac{\partial \epsilon_{\theta}}{\partial x_{j}}\right)
$$

and $c_{\epsilon_{\theta}}$ is a constant coefficient. The coefficients $c_{\epsilon_{\theta \theta_{1} s f s}}, c_{\epsilon_{\theta k_{1} s f s}}, c_{\epsilon_{\theta k_{2} s f s}}$ and $c_{\epsilon_{\theta \theta_{2} s f s}}$ appearing in Eq. (54) are given by Eqs. (29) and Eq. (84) for $P_{r} \approx 1$, Eq. (89) for $P_{r} \ll 1$ and Eq. (96) for $P_{r} \gg 1$. Figure 5 displays the variation of the coefficient $c_{\epsilon_{\theta \theta_{2}} s f_{s}}$ versus the location of the cutoff depending on

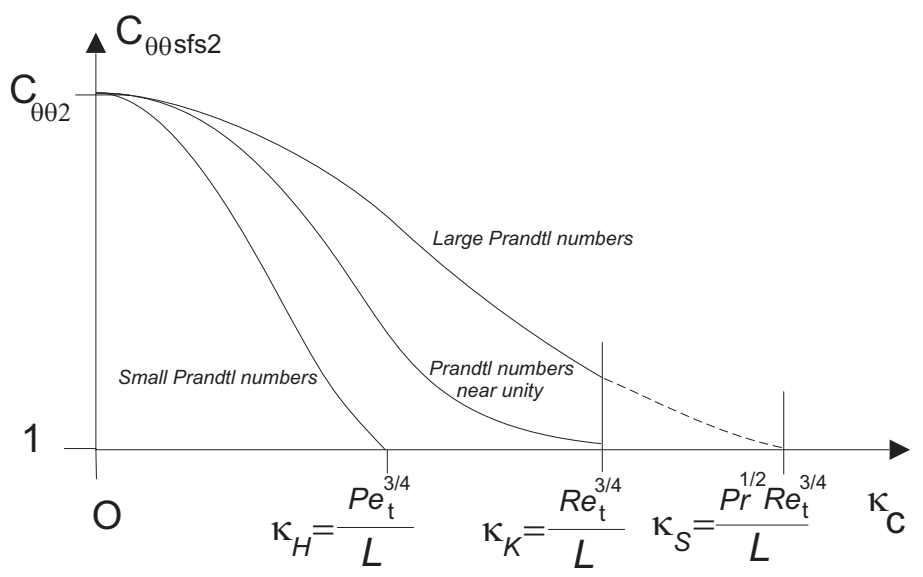

Figure 5: Qualitative sketch of the variation of the coefficient $c_{\epsilon_{\theta \theta_{2}}}$ versus location of cutoff depending on the Prandtl number ranges.

the Prandtl number ranges. As a result of interest, one can see that this coefficient varies between $c_{\epsilon_{\theta \theta_{2}}}$ and unity and that a plateau corresponding to $k_{\theta s f s} / k_{\theta}=1$ is obtained for a finite value of $\kappa_{c}$. So, the representative curve shows knee points which are $\kappa_{H}=P_{r}^{3 / 4} R e_{t}^{3 / 4} / L$ for small Prandtl numbers, 
$\kappa_{K}=R e_{t}^{3 / 4} / L$ for Prandtl number near unity and $\kappa_{S}=P_{r}^{1 / 2} R e_{t}^{3 / 4} / L$ for large Prandtl numbers (we recall that $L=k^{3 / 2} / \epsilon$ ), but continuity is always preserved. The dimensionless variables are recalled hereafter $\vartheta_{c}=\kappa_{c} k^{3 / 2} / \epsilon, \vartheta_{H}=R e_{t}^{3 / 4} P_{r}^{3 / 4}, \vartheta_{K}=R e_{t}^{3 / 4}, \vartheta_{S}=R e_{t}^{3 / 4} P_{r}^{1 / 2}$, respectively.

\subsection{Practical choice of the spectrum parameters and model coefficients}

As it was mentioned in section 4, the energy density spectrum is governed by the parameters $\alpha$ and $\gamma$. Trying to stick to the values suggested by the PITM study, we use here the values $c_{\epsilon_{\theta \theta_{1}}}=1, c_{\epsilon_{\theta k_{1}}}=1 / 2$, $c_{\epsilon_{\theta k_{2}}}=1 / 2$, and we choose $c_{\epsilon_{\theta \theta_{2}}}=1.30$. Note that there is no precise consensus in the scientific literature (Launder et al., 1984; Yoshizawa, 1988; Nagano and Kim, 1988; Ruffin et al., 1994; Schiestel, 2007; Hanjalic and Launder, 2011). With these numerical constants, the dimensionless number $\mathcal{R}$ takes on the value 0.75 . The coefficients values used in the turbulence model are summarized in table 1 . The

Table 2: Coefficients values used in the turbulence model.

\begin{tabular}{ccccccccccc}
\hline \hline$c_{\epsilon_{1}}$ & $c_{\epsilon_{2}}$ & $c_{k}$ & $c_{\epsilon}$ & $c_{\epsilon_{\theta \theta_{1}}}$ & $c_{\epsilon_{\theta k_{1}}}$ & $c_{\epsilon_{\theta k_{2}}}$ & $c_{\epsilon_{\theta \theta_{2}}}$ & $c_{\tau_{\theta}}$ & $c_{k_{\theta}}$ & $c_{\epsilon_{\theta}}$ \\
\hline 1.50 & 1.90 & 0.22 & 0.18 & 1 & 0.5 & 0.5 & 1.30 & 0.22 & 0.22 & 0.22 \\
\hline \hline
\end{tabular}

extension to low Reynolds number turbulence is considered in C for solving the viscous sublayer region.

\section{Application to the fully developed turbulent channel flow subjected to a constant heat flux}

\subsection{Filtered equations for the simulation in physical space}

The filtering of the instantaneous Navier-Stokes equations gives rise to the mass conservation and resolved scale motion equations as

$$
\begin{gathered}
\frac{\partial \bar{u}_{j}}{\partial x_{j}}=0 \\
\frac{\partial \bar{u}_{i}}{\partial t}+\frac{\partial\left(\bar{u}_{i} \bar{u}_{j}\right)}{\partial x_{j}}=-\frac{1}{\rho} \frac{\partial \bar{p}}{\partial x_{i}}+\nu \frac{\partial^{2} \bar{u}_{i}}{\partial x_{j} \partial x_{j}}-\frac{\partial\left(\tau_{i j}\right)_{s f s}}{\partial x_{j}}
\end{gathered}
$$


where $\left(\tau_{i j}\right)_{s f s}=\overline{u_{i} u_{j}}-\bar{u}_{i} \bar{u}_{j}$. In Eqs. (56) and (57), the commutation terms due to the fact that in the general case the filtering operation does not commute with the space or time derivative have been neglected (Chaouat and Schiestel, 2013; Chaouat, 2017c). The filtered transport equation for the passive scalar is

$$
\frac{\partial \bar{\theta}}{\partial t}+\frac{\partial\left(\bar{\theta} \bar{u}_{j}\right)}{\partial x_{j}}=\sigma \frac{\partial^{2} \bar{\theta}}{\partial x_{j} \partial x_{j}}-\frac{\partial\left(\tau_{j \theta}\right)_{s f s}}{\partial x_{j}}
$$

where $\left(\tau_{i \theta}\right)_{s f s}=\overline{u_{i} \theta}-\bar{u}_{i} \bar{\theta}$. This set of equation is solved to carry on the simulation. It is coupled with the PITM model to account for subfilter turbulence.

\subsection{Plane channel flow subjected to a constant heat flux on both walls}

This section aims to illustrate the theoretical developments accounting for passive scalar transport through basic numerical simulations. We perform the fully developed turbulent channel flow subjected to uniform heat fluxes $q_{w}$ at both walls as indicated in Fig. 6 for the Reynolds number $R_{\tau}=u_{\tau} \delta / \nu=395$ where $u_{\tau}$ denotes the friction velocity and $\delta$ is the half channel width, and for three values of the molecular Prandtl number $P_{r}=0.1,1$ and 10. Physically, these Prandtl numbers are chosen as representative of heat transfer in liquid metals such as mercury or liquid sodium $P_{r} \approx 0.015$ ), gases $\left(P_{r} \approx 1\right)$, water $\left(P_{r} \approx 5-7\right)$ and seawater $\left(P_{r} \approx 13\right.$ at $\left.0^{\circ} C\right)$. We have carried out a RANS-LES simulation based on PITM formalism of the turbulent plane channel flow in order to validate the subfilter model equations including both the dynamic equations and the thermal equations. In order to focus into the passive scalar field that constitutes the original part of this present work, the purely dynamical illustrative results are not presented here but they can be found in previous papers of the authors themselves (Chaouat and Schiestel 2005, 2009). The PITM simulations are then compared with reference DNS performed by Chaouat (2018), Chaouat and Peyret (2019).

\subsubsection{Numerical procedure}

The simulations are performed on several meshes of coarse and medium grid resolutions to study the grid effect on the scalar variable, turbulent heat fluxes $\left(\tau_{i \theta}\right)_{s f s}$, and variance of the passive scalar $k_{\theta}$ with emphasis placed on the sharing out between the modeled and resolved scales. The dimensions of the channel in the streamwise, spanwise and normal directions along the axes $x_{1}, x_{2}, x_{3}$ are respectively 


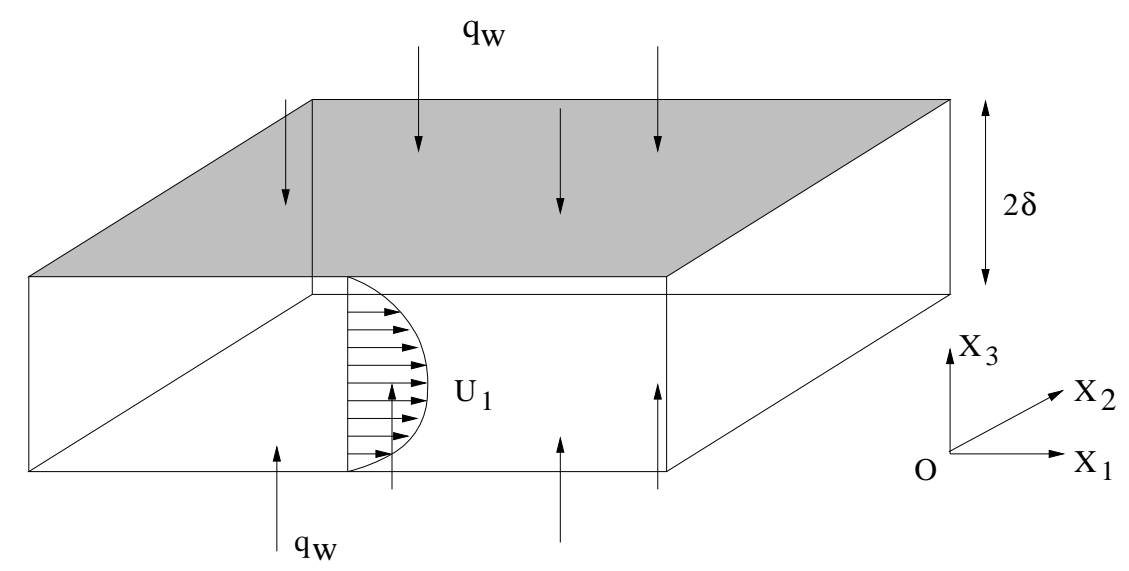

Figure 6: Setup of the numerical channel flow simulations with various thermal boundary conditions. Both walls are heated.

$L_{1}=6.4 \delta, L_{2}=3.2 \delta$ and $L_{3}=2 \delta$. The Batchelor length-scale is given by $\eta_{\theta}=\eta_{\kappa} / P_{r}^{3 / 4} \approx 5.62 \eta_{\kappa}$ at $P_{r}=0.1, \eta_{\theta} \approx \eta_{\kappa}$ at $P_{r}=1$, and $\eta_{\theta}=\eta_{\kappa} / P_{r}^{1 / 2} \approx 0.316 \eta_{\kappa}$ at $P_{r}=10$. For the Reynolds number and Prandtl number values studied here, the grids resolution in the $\left(x_{1}, x_{2}, x_{3}\right)$ directions as well and the grid spacings in wall unit are listed in Table 3. The meshes are uniform in the streamwise and spanwise directions $\left(x_{1}, x_{2}\right)$ and the grid spacings in wall unit are $\Delta_{1}^{+}=\Delta_{2}^{+}=60$ for the coarse mesh and $\Delta_{1}^{+}=\Delta_{2}^{+}=30$ for the medium mesh, respectively. The spacing in the center of the channel along the normal direction to the wall is $\Delta_{3 c}^{+}=16$. In this direction $x_{3}$, the grid points are distributed in different spacings with a refinement near the wall to compute accurately both the velocity and thermal boundary layers according to the transformation $x_{3 j}=\tanh \left[\xi_{j} F\left(\xi_{j}\right) \operatorname{atanh} a\right] / 2$ where $\xi_{j}=-1+2(j-1) /\left(N_{3}-1\right)\left(j=1,2, \cdots N_{3}\right), F\left(\xi_{j}\right)=\sqrt{\left(1+\xi_{j}^{2}\right) / 2}$, the parameter $a$ is a coefficient set to 0.990 for $N_{3}=84$ and 0.9875 for the $N_{3}=128$ leading to $\Delta_{3 w}^{+}=0.2$. For both cases, it is found that the larger grid spacing in the normal direction is then smaller than 16 in wall unit $\Delta_{3}^{+}<16$. This choice of grid size allows generally to get a significant part of the scalar variance in the subfilter part. However in the case of small Prandtl numbers, the vanishing wavenumber of the spectrum $\kappa_{H}$ is greatly diminished, inescapable clue of high scalar dissipation implying a very smaller subfilter part. But this is a physical consequence and does not mean at all that the grid is too fine. In terms of an order of resolution purely for information purposes, the grid-point and the spacings associated with the direct numerical 
simulation performed by Chaouat and Peyret (2019) are also indicated in Table 3. As a result of interest, the ratio of the DNS grid-points over the PITM grid-points $R\left(P_{r}\right)=N_{D N S} / N_{P I T M}$ varies from 113 for $P_{r}=0.1$ to 2378 for $P_{r}=10$. These values clearly show that all PITM grids are of coarse resolution in comparison with DNS grids and also highly resolved LES grids. It can be emphasized that the resolution of the grid for a direct numerical simulation of scalar turbulence is loose at low Prandtl number but fine at large Prandtl number according to the power law of the Prandtl number $N \propto R_{t}^{9 / 4} P_{r}^{3 / 2}$ where $R_{t}=k^{2} /(\nu \epsilon)$ is the turbulent Reynolds number, because the thermal scales are smaller than the dynamic Kolmogorov scales. At $P_{r}=1, R_{2}\left(P_{r}\right)=N_{D N S} / N_{P I T M 2}=451$ and $R_{1}\left(P_{r}\right)=N_{D N S} / N_{P I T M 1}=113$ while at $P_{r}=10, R_{2}\left(P_{r}\right)=N_{D N S} / N_{P I T M 2}=2378$ and $R_{1}\left(P_{r}\right)=N_{D N S} / N_{P I T M 1}=594$. This means that the present grids used here, especially at the Prandtl number $P_{r}=10$ are in fact very coarsened in comparison with the DNS grids for such PITM simulations that run in LES mode far away the walls because much more scales need to be modeled. The simulations are performed using the numerical code developed by Chaouat (2011) which is based on the finite volume technique and optimized with message passing interface (MPI). The equations are integrated in time by means of an implicit Runge-Kutta scheme of fourth-order accuracy in time. The Navier-Stokes equations are solved in space by a centered scheme of fourth-order accuracy in space whereas the transport equations of turbulence and scalar fields are solved by an upwind scheme of second-order accuracy in space. Equations (58) and (52) associated with the mean scalar variable are independent from the set of Eqs. (50) and (54) associated with the scalar variance. A constant pressure gradient term is included in the momentum equation to balance the viscous effects at the walls and an extra source term is added in the transport equation of the scalar variable aiming to get a periodic condition between the inlet and outlet plane sections of the channel (Chaouat, 2018; Chaouat and Peyret, 2019). The boundary conditions imposed at the lower and upper walls located at $x_{3}=0$ and $2 \delta$ of the channel are no slip velocity conditions $\bar{u}_{i}=0$. A constant heat flux corresponding to an isoflux boundary condition given by $q_{w}=-\kappa\left(\partial \bar{\theta} / \partial x_{3}\right)_{w}$ is applied at the upper and lower walls.

\subsubsection{Numerical results}

As usually, the results are presented in dimensionless form using the half channel width $\delta$, the wall friction velocity $u_{\tau}$ and the friction scalar variable $\theta_{w}=q_{w} /\left(\rho c_{p} u_{\tau}\right)$ where $c_{p}$ denotes the specific heat at 
constant pressure. The transformed variable $\Theta^{+}=\theta_{w}^{+}-\theta^{+}$is considered in order to analyze the present results because the mean statistical quantities including the velocity and normal turbulent stresses are symmetric about the centerline of the channel. Figure 7 describes the profile of the mean scalar variable $\left\langle\Theta^{+}\right\rangle$versus the logarithmic wall distance for several Prandtl numbers returned by the two simulations performed on the coarse and medium meshes, where the brackets $\langle$.$\rangle denotes the averaging$ in homogeneous directions of the flow. As known, the logarithmic region gets more extended when the Prandtl number increases from 0.1 to 10. An excellent agreement is observed between the DNS and PITM simulations at each Prandtl number confirming that the thermal boundary layer is accurately resolved in the wall region. As a preliminary requirement, it is necessary to verify the predictions of the turbulent heat fluxes. Indeed these turbulent fluxes not only control the mean distribution of temperature but also appear in the driving source term of the variance, they are thus twice important. Figures 8 and 9 exhibit the profiles of the streamwise and normal turbulent heat fluxes $\left|q_{1}\right|=\left\langle u_{1}^{\prime+} \theta^{\prime+}\right\rangle$ and $q_{3}=\left\langle u_{3}^{\prime+} \theta^{\prime+}\right\rangle$, respectively, versus the wall distance for the Prandtl numbers $P_{r}=0.1,1$ and 10 and for the two simulations performed on the coarse and medium meshes. Overall, it is found that the PITM simulation provides turbulent heat fluxes in good agreement with the reference DNS data. The intensity of the streamwise turbulent heat flux for the Prandtl number $P_{r}=0.1$ and unity appears slightly overpredicted when moving towards the center of the channel because of the resolved contribution which decreases a little bit too slowly away from the walls. This is probably due to the coarse grid resolution in this flow region. As expected, the normal turbulent heat flux is accurately predicted for each Prandtl number according to the DNS data. These figures show that the subfilter turbulent heat fluxes are relatively high in the near wall region revealing the presence of a thermal peak for $P_{r}=1$ and 10 followed afterward by a rapid decrease when going away from the wall, while the resolved turbulent heat fluxes increase and decrease more slowly versus the wall distance. The curves associated with the resolved turbulent heat fluxes present a regular evolution from the wall to the centerline of the channel. Because of the grid resolution that has been chosen, the subfilter part of the turbulent heat flux as determined by the location of the cutoff wave number $\kappa_{c}$ in the spectrum of the scalar variable $E_{\theta}(\kappa)$ appears of lower intensity than its corresponding resolved part almost everywhere in the channel even if the sharing out of the energy is appreciably modified with respect to the Prandtl number. Indeed, as the Prandtl number increases from the lower value $P_{r}=0.1$ to the 
higher value 10, the subfilter part is increasing relatively to its resolved part and conversely, the resolved part is decreasing. This is an important point suggesting that the model is consistent between the two extremes limits of the energy spectra splittings of $E(\kappa)$ and $E_{\theta}(\kappa)$ that are RANS and DNS. As expected, it is found that the subfilter heat flux energy increases as the grid-size increases since larger scales must be modeled and vice versa, the resolved heat flux energy decreases since less scales are computed. But as a remarkable result, it is found that the sum of each contribution, modeled and resolved parts for the coarse and medium corresponds to the DNS fairly well. This observation appears so clear for the normal turbulent heat fluxes $q_{3}=\left\langle u_{3}^{\prime+} \theta^{\prime+}\right\rangle$ but is less marked for the streamwise turbulent heat flux $q_{1}=\left\langle u_{1}^{\prime+} \theta^{\prime+}\right\rangle$. Hence, the analysis of the results with respect the two grids considered confirms that the PITM method returns heat fluxes in good agreement with DNS independently of the spectral cutoff location. It is worth mentioning that the mean scalar variable $\langle\theta\rangle$ deduced from the instantaneous filtered variable $\bar{\theta}$ solution of Eq. (58) is here governed by the normal subfilter turbulent heat $\left(\tau_{3 \theta}\right)_{s f s}$. As the mean scalar profile shown in Fig. 7 is fairly well predicted from the simulation at each Prandtl number, it is not at all surprising that the normal heat flux profiles agree very well with the DNS data for all grids. So, this outcomes demonstrates that the closure (52) used for the turbulent heat flux assuming the generalized gradient diffusion hypothesis GGDH [21] is entirely satisfactory for solving the scalar-velocity correlations in the present application. This one is generally successful in heat transfer of practical engineering and geophysical flows when the Reynolds stress anisotropy is reasonably well captured both in RANS and LES (Launder, 1988; Suga, 2004; Kenjeres and Hanjalic, 2006; Kenjeres et al., 2015). As regards temperature variance predictions, figure 10 displays the root-mean square of the scalar variance $\theta_{r m s}^{+}=\sqrt{\left\langle\theta^{\prime+} \theta^{\prime+}\right\rangle}=\sqrt{2 k_{\theta}}$ computed by means of the subgrid and resolved fluctuations as follows

$$
\left\langle\theta^{\prime} \theta^{\prime}\right\rangle=[\langle\overline{\theta \theta}\rangle-\langle\bar{\theta} \bar{\theta}\rangle]+[\langle\bar{\theta} \bar{\theta}\rangle-\langle\theta\rangle\langle\theta\rangle]=2\left(k_{\theta s f s}+k_{\theta l e s}\right)
$$

respectively, for the simulations performed on the coarse and medium meshes. A quantitative agreement is obtained with the DNS although the PITM is performed on a relatively coarse grid, especially in the streamwise and spanwise directions of the mesh. For $\operatorname{Pr}=0.1$, the subfilter part of the scalar variance appears to be of lower intensity than the resolved part everywhere in the channel. This result is not a shortcoming but must be attributed to Eqs. (84), (89) and (96) accounting for the dimensionless numbers $\vartheta_{c}, \vartheta_{H}$ and $\vartheta_{K}$. Indeed at very low Prandtl numbers, the variance spectrum is falling down 
very early and thus contains very few small eddies of the scalar. In other words, the high diffusivity efficiently cancel these small eddies. As has been observed previously for the turbulent heat fluxes, when the grid-size increases in space $\partial \Delta\left(x_{i}\right) / \partial x_{i}>0$, then a part of energy $k_{\theta}$ contained into the resolved scales is removed and fed into the modeled spectral zone, whereas on the contrary, when $\partial \Delta\left(x_{i}\right) / \partial x_{i}<0$, a part of energy coming from the modeled zone is injected into the resolved scales but the total variance $k_{\theta}$ remains almost the same for the two grids considered as it was also obtained for the turbulence energy $k$ (Chaouat and Schiestel 2005, 2012; Chaouat, 2017c). Figure 11 shows the contours plots of the instantaneous filtered scalar field $\Theta$ for the Prandtl number $P_{r}=1$ in the $\left(x_{1}, x_{3}\right)$ mid-plane of the channel. The unsteady character of the scalar field is well visible. In particular, this figure puts in light the detachment of vortex in the normal direction to the wall according to the direct numerical simulation performed by Chaouat and Peyret, 2019 (see Figure 14) even if some details are lost in the present case because of the very coarse grid resolution. In this context, it can be recalled that RANS computations cannot reproduce instantaneous structures in time and space because of the RANS physical foundations relying in practice on a long-time averaging which is large in comparison with the turbulence time scale (Chaouat, 2017). As known, RANS determine only mean organized structures as for instance the parietal vortex shedding that develop in a channel flow with wall mass injection (Chaouat and Schiestel, 2002). 
Table 3: Simulation parameters for the fully developed turbulent channel flow, grid-points $N_{i}$, total number of grid-points $N$, grid resolution $\Delta_{i}^{+}$, in the streamwise, spanwise and wall-normal directions, $x_{1}, x_{2}, x_{3}$, respectively, and Batchelor length-scale $\eta_{\theta}$ relatively to the Kolmogorov length-scale $\eta_{\kappa}$ for the Prandtl numbers $P_{r}=0.1,1$ and 10. Domain size $L_{1} \times L_{2} \times L_{3}=6.4 \delta \times 3.2 \delta \times 2 \delta$. Present PITM simulations performed at the Reynolds number $R_{\tau}=u_{\tau} \delta / \nu=395$. DNS (Chaouat and Peyret, 2019).

\begin{tabular}{|ccccccccc|}
\hline Case & $N_{1}$ & $N_{2}$ & $N_{3}$ & $N\left(10^{6}\right)$ & $\Delta_{1}^{+}$ & $\Delta_{2}^{+}$ & $\Delta_{3 c}^{+}$ & $\eta_{\theta}$ \\
\hline DNS $(\operatorname{Pr}=0.1)$ & 256 & 128 & 256 & 8.38 & 10 & 10 & 5.1 & $5.62 \eta_{\kappa}$ \\
\hline DNS $(\operatorname{Pr}=1)$ & 512 & 256 & 256 & 33.55 & 5 & 5 & 5.1 & $\eta_{\kappa}$ \\
\hline DNS $(\operatorname{Pr}=10)$ & 1024 & 512 & 512 & 268.44 & 2.5 & 2.5 & 2.2 & $0.316 \eta_{\kappa}$ \\
\hline PITM1 $(\operatorname{Pr}=0.1)$ & 42 & 21 & 84 & 0.074 & 60 & 60 & 16 & $5.62 \eta_{\kappa}$ \\
\hline PITM1 $(\operatorname{Pr}=1)$ & 42 & 21 & 84 & 0.074 & 60 & 60 & 16 & $\eta_{\kappa}$ \\
\hline PITM1 $(\operatorname{Pr}=10)$ & 42 & 21 & 128 & 0.112 & 60 & 60 & 16 & $0.316 \eta_{\kappa}$ \\
\hline PITM2 $(\operatorname{Pr}=0.1)$ & 84 & 42 & 84 & 0.296 & 30 & 30 & 16 & $5.62 \eta_{\kappa}$ \\
\hline PITM2 $(\operatorname{Pr}=1)$ & 84 & 42 & 84 & 0.296 & 30 & 30 & 16 & $\eta_{\kappa}$ \\
\hline PITM2 $(\operatorname{Pr}=10)$ & 84 & 42 & 128 & 0.452 & 30 & 30 & 16 & $0.316 \eta_{\kappa}$ \\
\hline
\end{tabular}




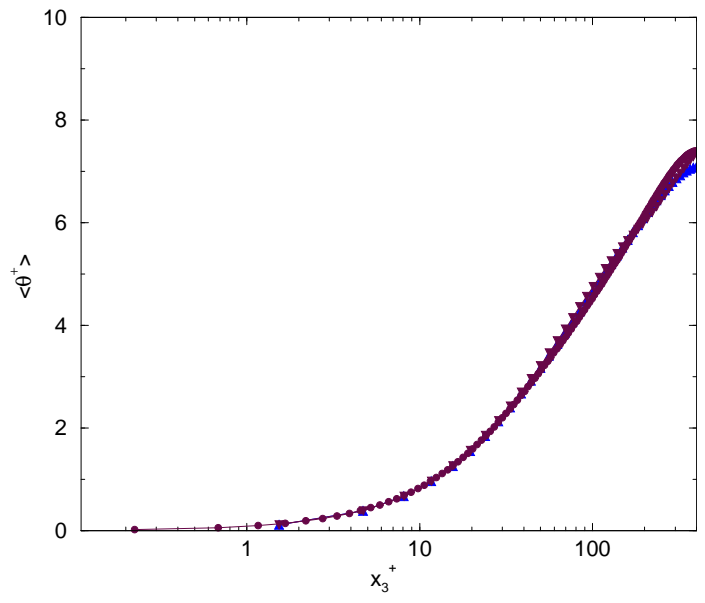

(a)

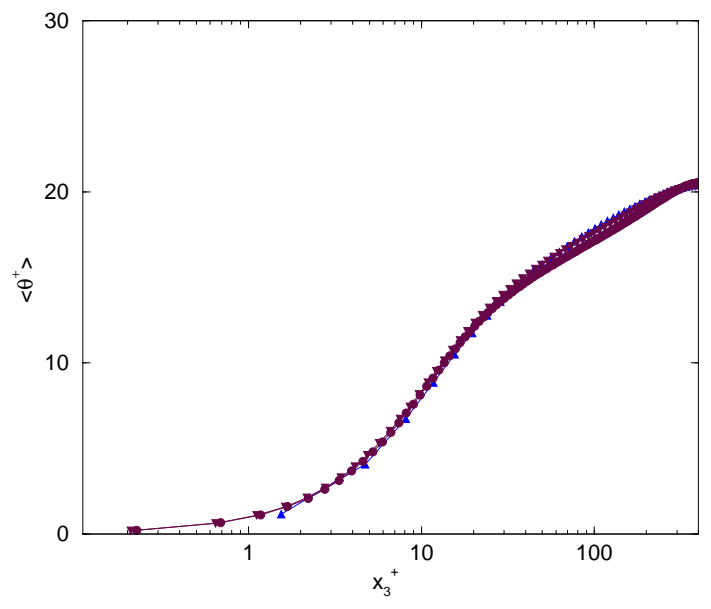

(b)

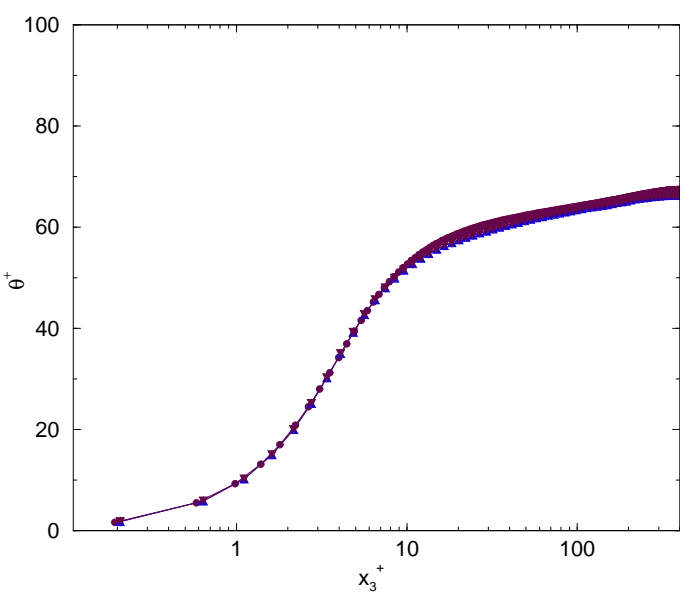

(c)

Figure 7: Mean scalar field $\left\langle\theta^{+}\right\rangle=\langle\theta\rangle / \theta_{\tau}$ in logarithmic coordinate versus the wall unit distance for various $P_{r}$ numbers. DNS : •; PITM1: $\nabla$. PITM2: $\boldsymbol{\Delta}$. (a) $P_{r}=0.1$; (b) $P_{r}=1$; (c) $P_{r}=10 ; R_{\tau}=395$. 

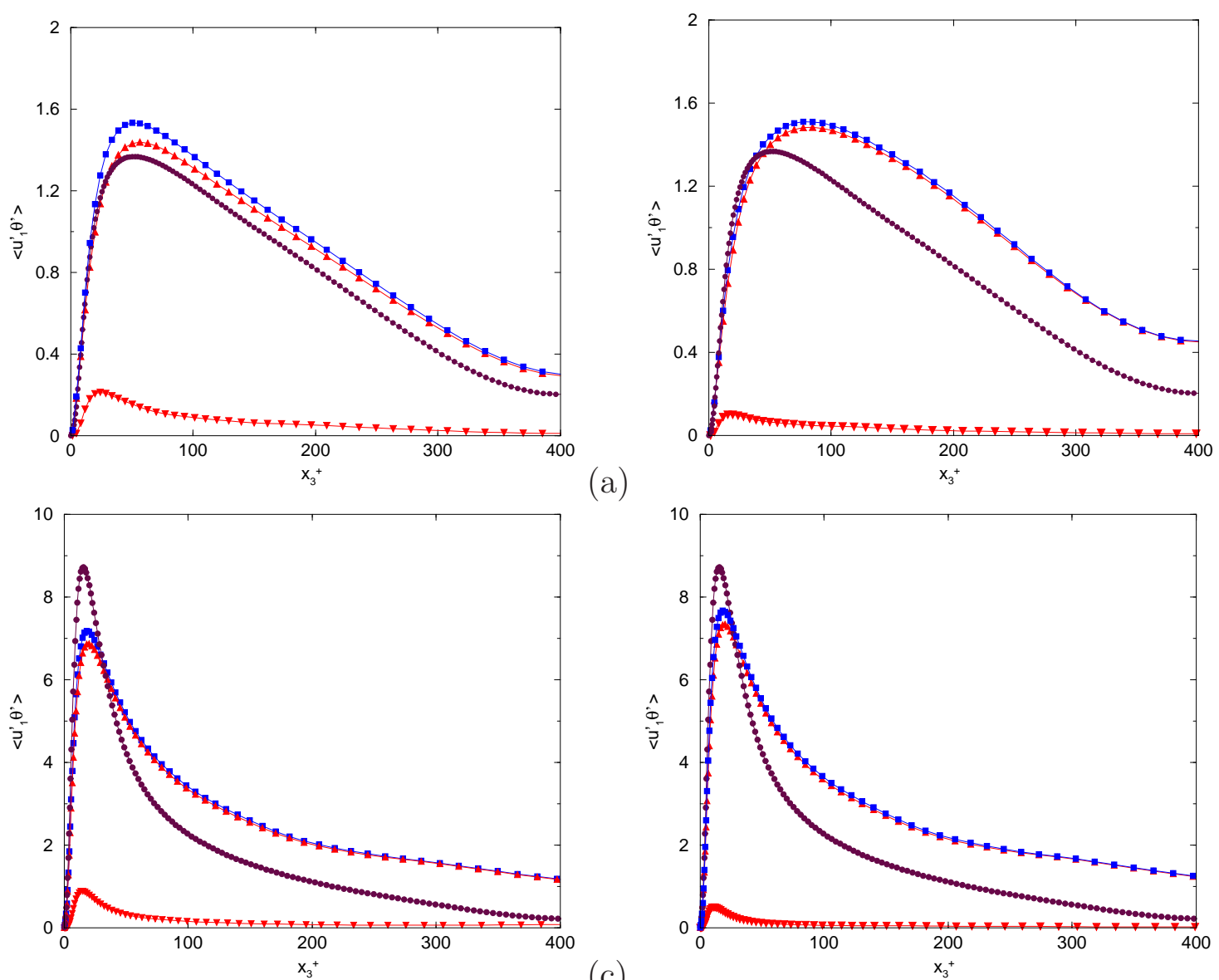

(a)

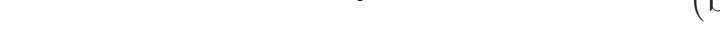

(c)

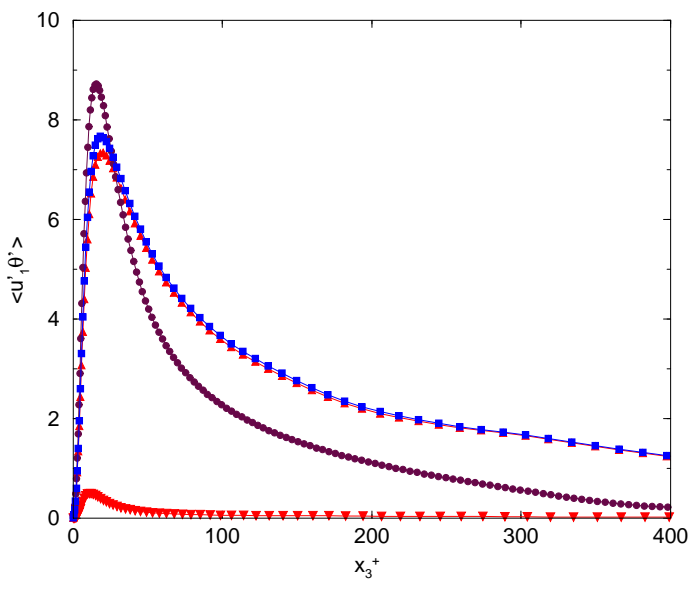

(d)
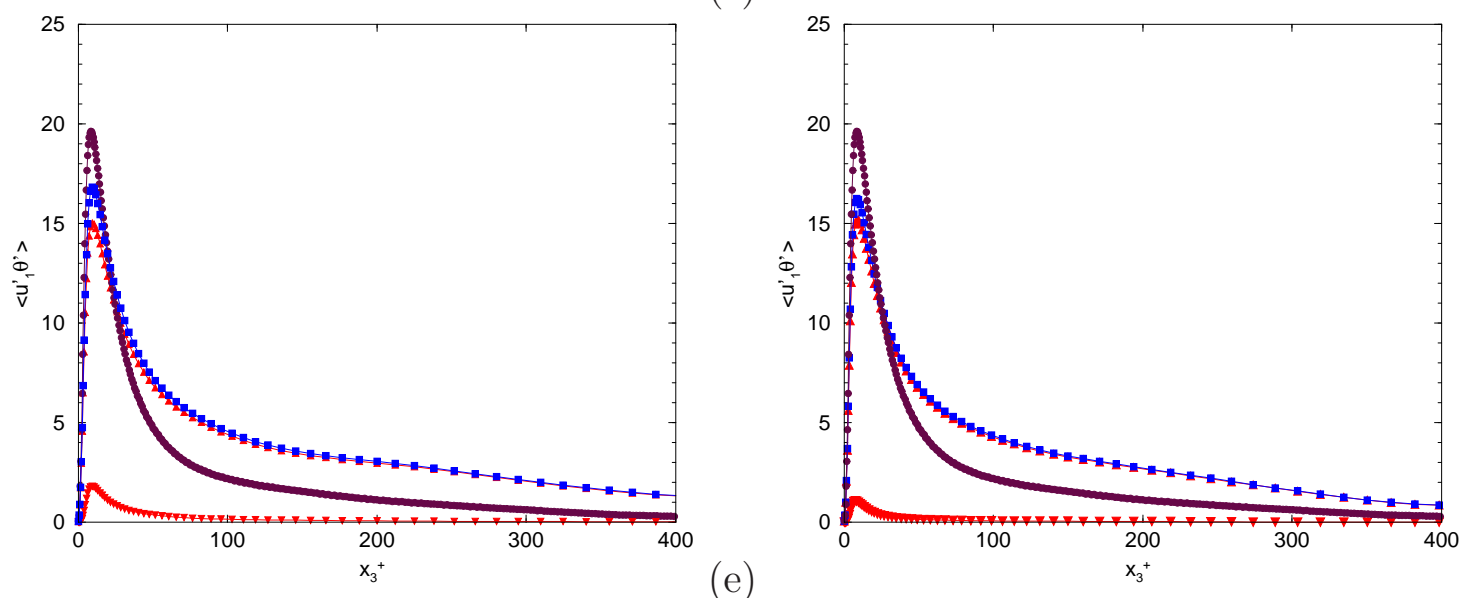

Figure 8: Streamwise turbulent heat fluxe $\left|q_{1}\right|=\left\langle u_{1}^{\prime+} \theta^{\prime+}\right\rangle=\left\langle u_{1}^{\prime} \theta^{\prime}\right\rangle / u_{\tau} \theta_{\tau}$ versus the wall distance for various Prandtl numbers. (a), (b): $P_{r}=0.1$. (c), (d): $P_{r}=1$. (e), (f): $P_{r}=10$. DNS : •; Left : PITM1: Right: PITM2. Subfilter scale : $\mathbf{\nabla}$; Resolved scale : $\mathbf{\Delta}$; Total scales : 

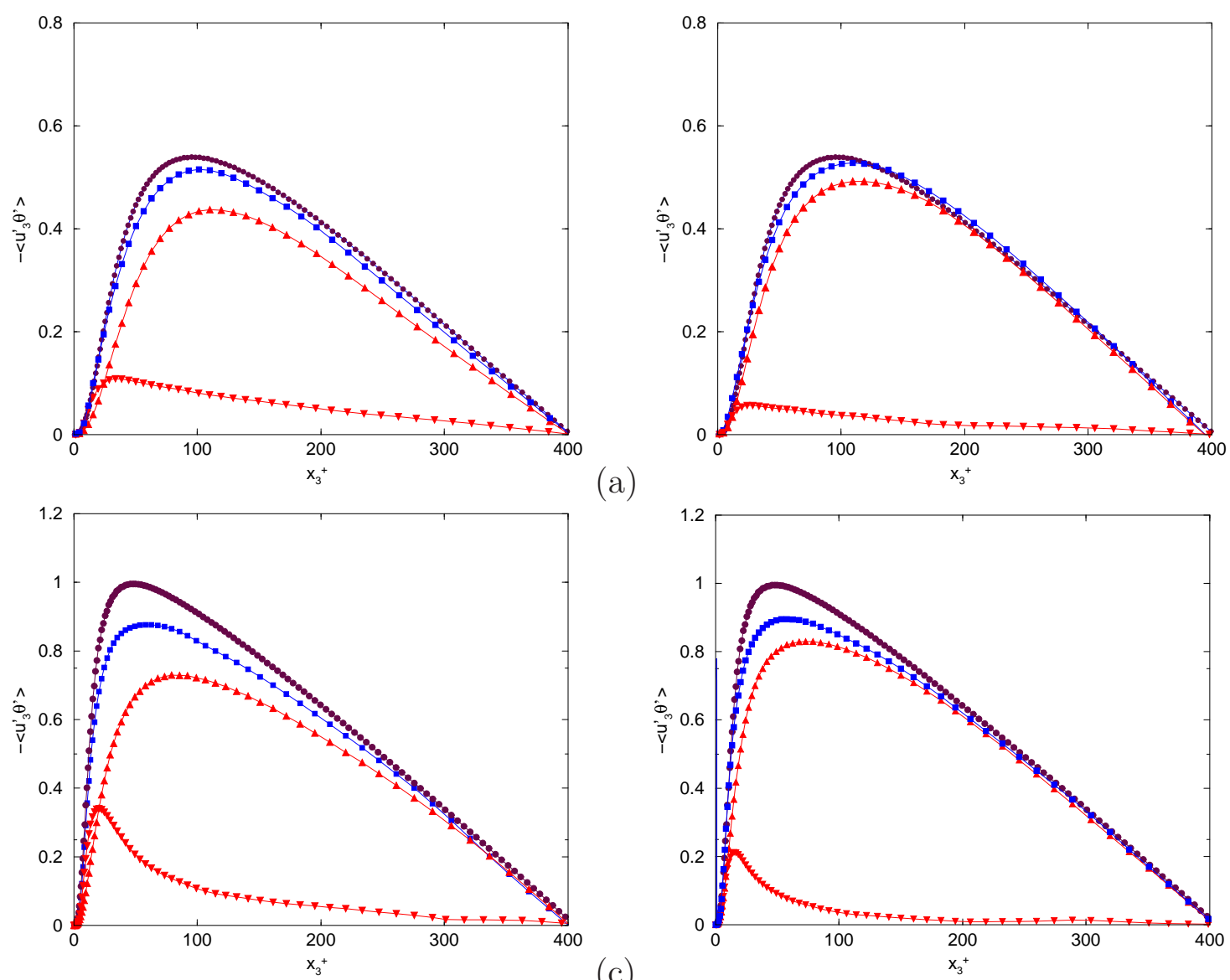

(a)

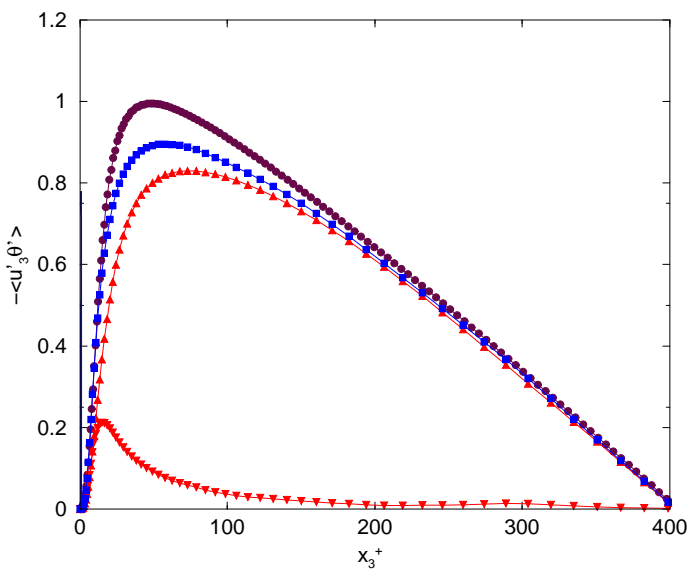

(c)
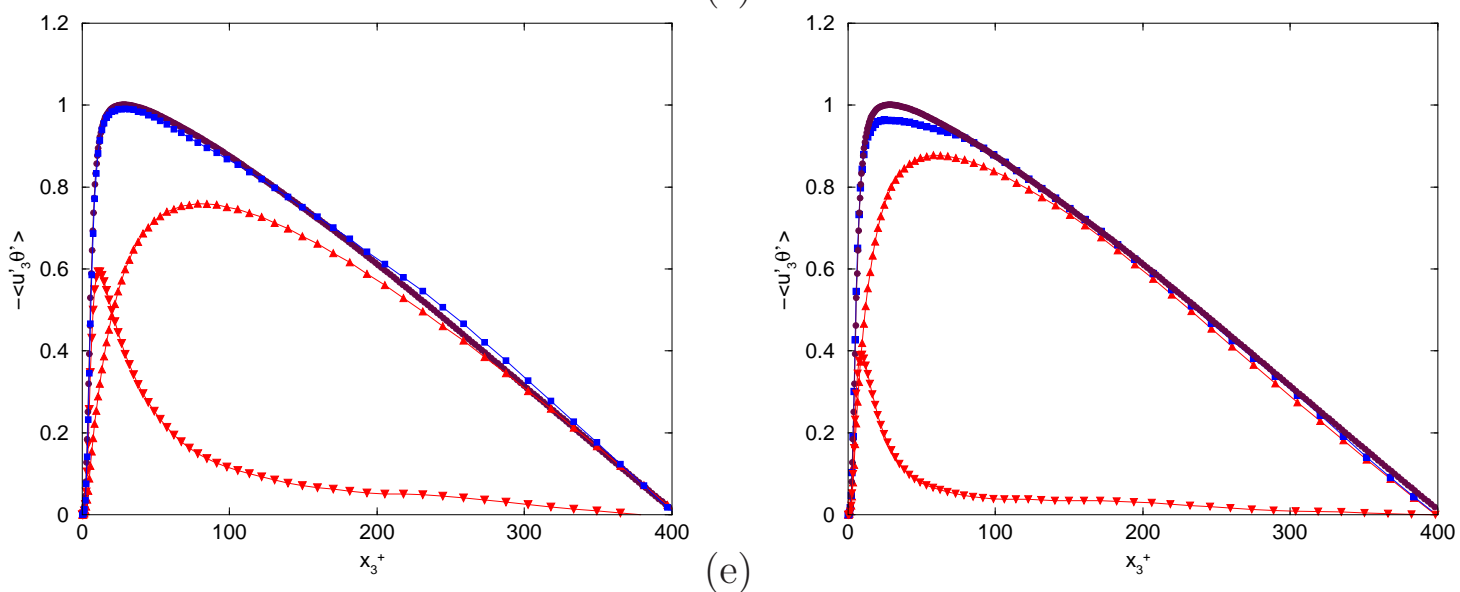

(e)

Figure 9: Normal turbulent heat fluxes $q_{3}=\left\langle u_{3}^{\prime+} \theta^{\prime+}\right\rangle=\left\langle u_{3}^{\prime} \theta^{\prime}\right\rangle / u_{\tau} \theta_{\tau}$, versus the wall distance for various Prandtl numbers. (a), (b): $P_{r}=0.1$. (c), (d): $P_{r}=1$. (e), (f): $P_{r}=10$. DNS : •; Left : PITM1: Right: PITM2. Subfilter scale : $\mathbf{\nabla}$; Resolved scale : $\mathbf{\Delta}$; Total scales : 

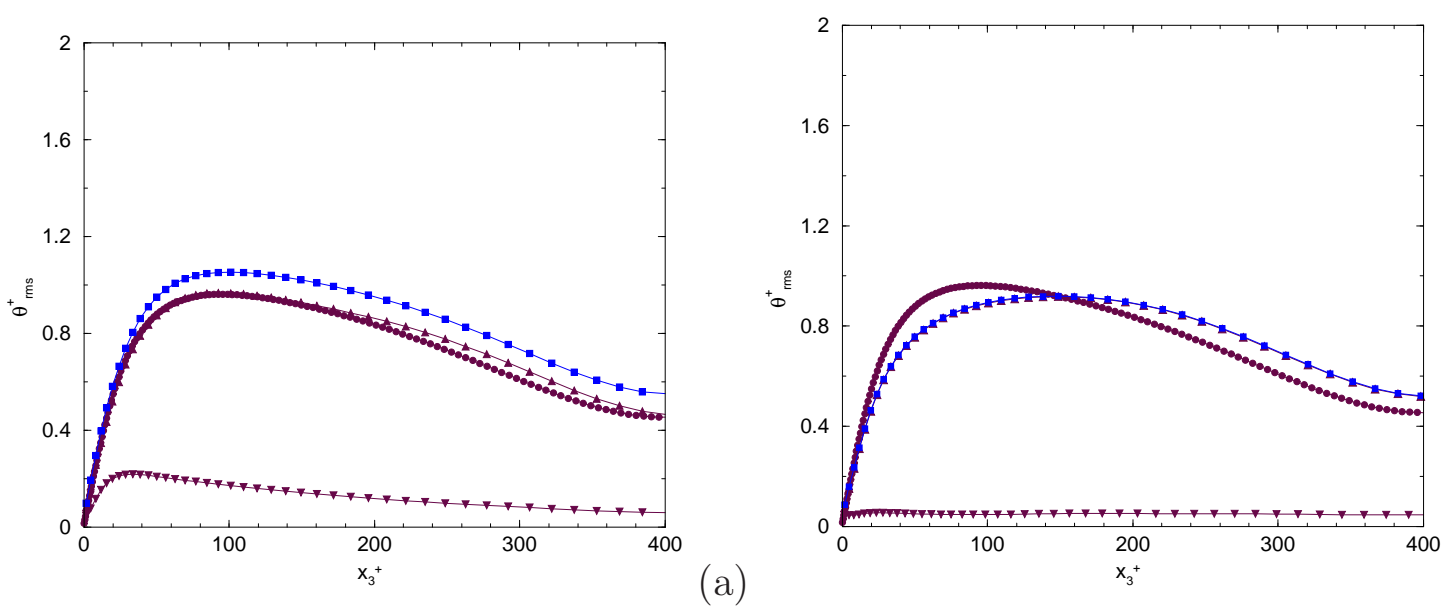

(b)
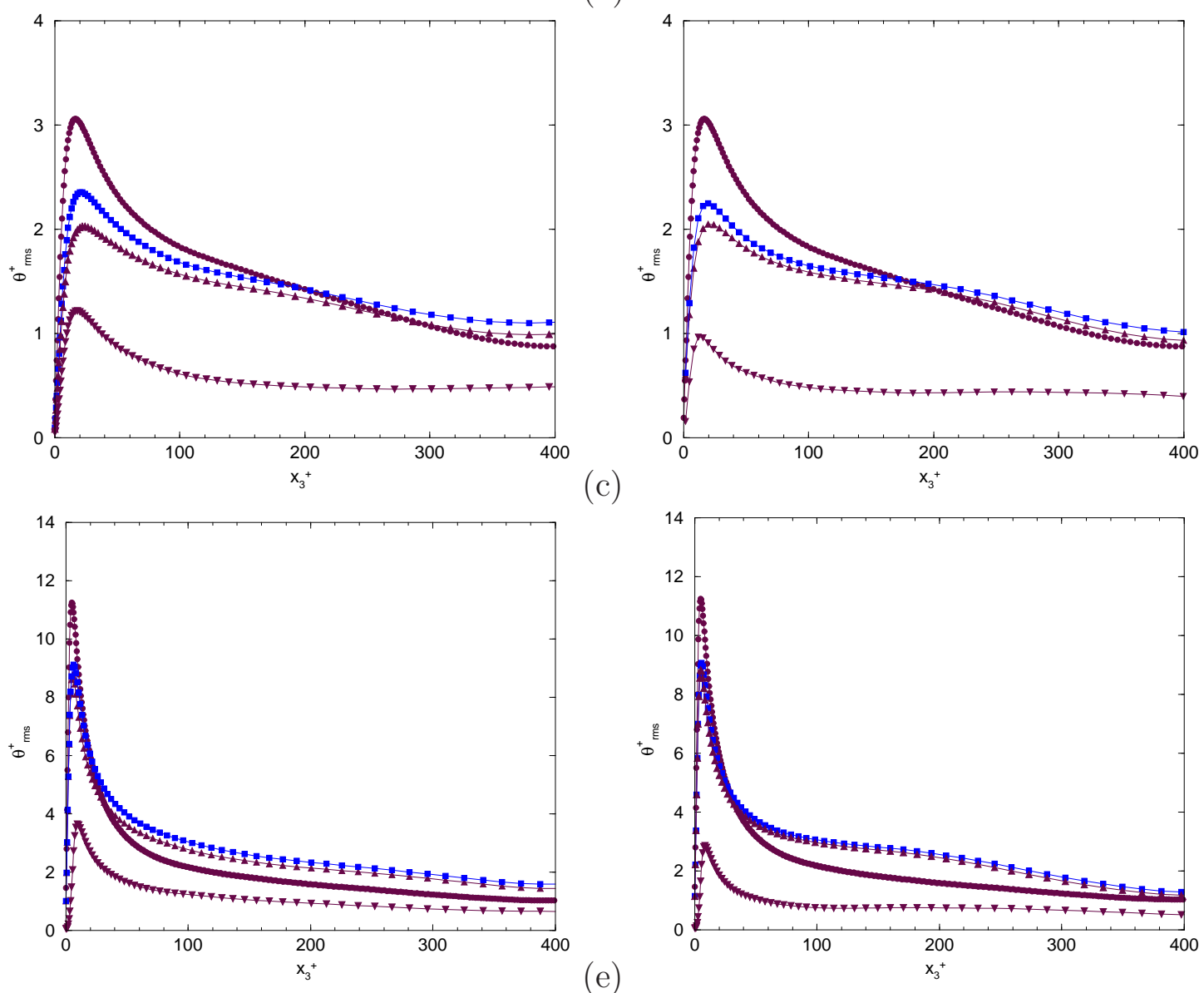

(f)

Figure 10: Root mean square of the scalar variance $\theta_{r m s}^{+}=\sqrt{\left\langle\theta^{\prime+} \theta^{\prime+}\right\rangle}=\sqrt{\left\langle\theta^{\prime} \theta^{\prime}\right\rangle} / \theta_{\tau}$ versus the wall distance for various $P_{r}$ numbers. (a),(b) $P_{r}=0.1$; (b),(c) $P_{r}=1$; (c),(d) $P_{r}=10$; Left : PITM1; Right: PITM2. DNS : •; Subfilter scale : $\mathbf{\nabla}$; Resolved scale : $\boldsymbol{\Delta}$. Total scales : $\bullet . R_{\tau}=395$. 


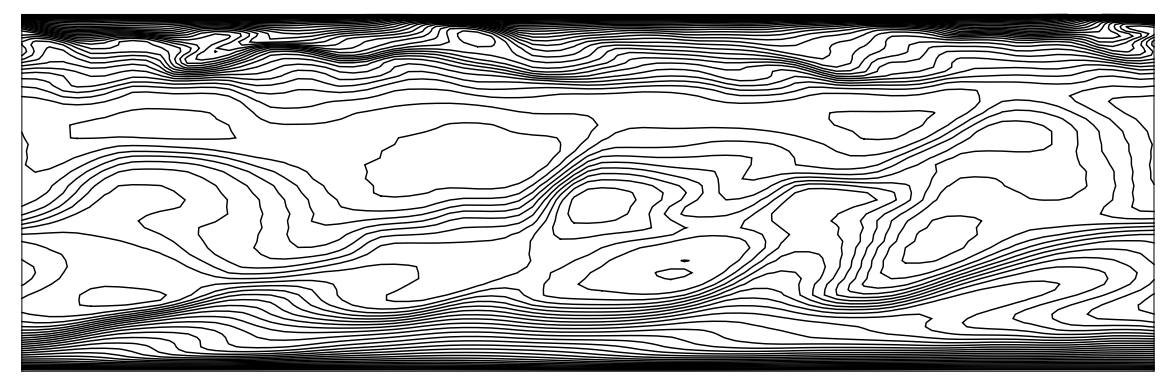

Figure 11: Contours of the instantaneous filtered variable $\Theta$ in the $\left(x_{1}, x_{3}\right)$ mid-plane illustrating the unsteady character of the scalar field. PITM2. Comparison can be made with DNS (Chaouat and Peyret, 2019).

$$
P_{r}=1 ; R_{\tau}=395
$$

\section{$7 \quad$ Concluding remarks}

The present work was devoted to the extension of the PITM method initially developed for simulating unsteady turbulent flows to the associated passive scalar transport fields from a physical and analytical standpoint. Emphasis has been put on the methodology. In this framework, we make use of the concept of tangent homogeneous space to derive the spectral transport equation for the variance of the passive scalar. Using partial integration in the spectral space and spectral splitting techniques, we have then derived the equations for the subfilter variance $k_{\theta s f s}$ and its dissipation-rate $\epsilon_{\theta}$ in the physical space. As a result, it appears that the dissipation-rate equation for $\epsilon_{\theta}$ takes the same form as the usual corresponding RANS equation but the coefficient $c_{\theta \theta s f s_{2}}$ appearing in the destruction term is now a function of the ratio of the subfilter variance to the total variance $k_{\theta s f s} / k_{\theta}$ taking into account the cutoff wave number $\kappa_{c}$ and the turbulence length scale $L=k^{3 / 2} / \epsilon$. Considering different spectra $E_{\theta}(\kappa)$ of the passive scalar depending on the Prandtl number range, the mathematical expression of the subfilter coefficient has been calculated as a function of the dimensionless variables involving the characteristic length-scales, the Prandtl number and the Peclet number. These foundation concepts in spectral space 
have then be used to develop the subfilter closure model extended to turbulent scalar fluctuations. In a first step, we have then considered for the turbulent field the second moment closure based on the transport equations of the subfilter scale stresses $\left(\tau_{i j}\right)_{s f s}$ and the dissipation-rate $\epsilon$, coupled with an algebraic diffusivity model for the scalar fluxes $\left(\tau_{i \theta}\right)_{s f s}$ used in the transport equations of the variance of the subfilter scalar field $k_{\theta s f s}$ and its dissipation-rate $\epsilon_{\theta}$. Numerical simulations of fully turbulent channel flows have been performed on several grids for illustrating the capabilities of the present PITM model to reproduce passive scalar fields. Considering that a good prediction of turbulent scalar fluxes is a prerequisite for meaningful scalar variance study, thorough comparisons of heat fluxes with DNS data have been detailed with satisfactory results. The PITM yields these scalar field correlations in good agreement with DNS data for both grids. It has been verified that the subfilter contributions increase as the grid-size increases since larger scales must be modeled and vice versa, the resolved contributions decrease since less scales are computed. But as expected, the PITM model returns the total turbulent heat flux and scalar variance as free of any spectral cutoff location proving the entire self-consistency of the model. Moreover, some insights into the structural appearance of the scalar field interpreted as a marker for the fluid particles has been given. From a more general point of view, this pioneering work opens new routes of modeling for the simulation of turbulent flows including a passive scalar with a drastic reduction of the computational time and memory in term of number of grid points, in comparison with the demanding resources of highly resolved LES. Applications to practical and geophysical flows will be tackled in further works.

\section{A TRANSPORT EQUATION OF THE TWO POINT SCALAR FLUCTUATION}

\section{A.1 Transport equation of the two-point correlation tensor}

The instantaneous transport equation for a passive scalar can be written as

$$
\frac{\partial \theta}{\partial t}+u_{j} \frac{\partial \theta}{\partial x_{j}}=\sigma \frac{\partial^{2} \theta}{\partial x_{j} \partial x_{j}}
$$


where $\sigma=\lambda / \rho c_{p}=\nu / P_{r}$ is the thermal diffusivity, $\lambda$ is the thermal conductivity, $c_{p}$ is the specific heat at constant pressure, $\rho$ is the density, $P_{r}$ is the Prandtl number and $\nu$ denotes the molecular viscosity. Using the decomposition $\theta=\langle\theta\rangle+\theta^{\prime}$, the transport equation for the fluctuating temperature $\theta^{\prime}$ is then

$$
\frac{\partial \theta^{\prime}}{\partial t}+\left\langle u_{j}\right\rangle \frac{\partial \theta^{\prime}}{\partial x_{j}}=-u_{j}^{\prime} \frac{\partial\langle\theta\rangle}{\partial x_{j}}-u_{j}^{\prime} \frac{\partial \theta^{\prime}}{\partial x_{j}}+\sigma \frac{\partial^{2} \theta^{\prime}}{\partial x_{j} \partial x_{j}}+\frac{\partial}{\partial x_{j}}\left\langle u_{j}^{\prime} \theta^{\prime}\right\rangle
$$

We consider the general case of nonisotropic inhomogeneous turbulence. In this case, the two-point temperature correlation $\phi_{\theta \theta}=\left\langle\theta_{A}^{\prime} \theta_{B}^{\prime}\right\rangle\left(\boldsymbol{x}_{A}, \boldsymbol{x}_{B}\right)$, where $\theta_{A}^{\prime}$ is treated as a function of $\boldsymbol{x}_{A}$ whereas $\theta_{B}^{\prime}$ is a function of $\boldsymbol{x}_{B}$, considering that $\boldsymbol{x}_{A}$ and $\boldsymbol{x}_{B}$ are the primary independent variables. The transport equation for $\phi_{\theta \theta}$ is

$$
\begin{aligned}
& \frac{\partial \phi_{\theta \theta}}{\partial t}+\left\langle u_{j A}\right\rangle \frac{\partial \phi_{\theta \theta}}{\partial x_{j A}}+\left\langle u_{j B}\right\rangle \frac{\partial \phi_{\theta \theta}}{\partial x_{j B}}=-\left\langle u_{j A}^{\prime} \theta_{B}^{\prime}\right\rangle \frac{\partial\left\langle\theta_{A}\right\rangle}{\partial x_{j A}}-\left\langle\theta_{A}^{\prime} u_{j B}^{\prime}\right\rangle \frac{\partial\left\langle\theta_{B}\right\rangle}{\partial x_{j B}} \\
& -\frac{\partial}{\partial x_{j A}}\left\langle\theta_{A}^{\prime} u_{j A}^{\prime} \theta_{B}^{\prime}\right\rangle-\frac{\partial}{\partial x_{j B}}\left\langle\theta_{A}^{\prime} u_{j B}^{\prime} \theta_{B}^{\prime}\right\rangle \\
& +\sigma\left(\frac{\partial^{2} \phi_{\theta \theta}}{\partial x_{j A} \partial x_{j A}}+\frac{\partial^{2} \phi_{\theta \theta}}{\partial x_{j B} \partial x_{j B}}\right)
\end{aligned}
$$

New independent variables defined by the vector difference $\boldsymbol{\xi}=\boldsymbol{x}_{B}-\boldsymbol{x}_{A}$ and the midway position $\boldsymbol{X}=\frac{1}{2}\left(\boldsymbol{x}_{A}+\boldsymbol{x}_{B}\right)$ are then introduced in the present derivation in order to distinguish the effects of distance separation from the effects of space location. So that each variable can be regarded as a new function of the two variables $\boldsymbol{\xi}$ and $\boldsymbol{X}$. The formula for derivatives in the change of variables are detailed in Ref. [29], the main ones being

$$
\left(\frac{\partial}{\partial x_{k}}\right)_{A}=\frac{1}{2} \frac{\partial}{\partial X_{k}}-\frac{\partial}{\partial \xi_{k}}
$$

and

$$
\left(\frac{\partial}{\partial x_{k}}\right)_{B}=\frac{1}{2} \frac{\partial}{\partial X_{k}}+\frac{\partial}{\partial \xi_{k}}
$$

We introduce also the tensors $\phi_{j \theta}=\left\langle u_{j A}^{\prime} \theta_{B}^{\prime}\right\rangle$ and $\phi_{\theta j}=\left\langle\theta_{A}^{\prime} u_{j B}^{\prime}\right\rangle$. Taking into account these considerations, the complete dynamic equation (62) for the double temperature correlation for incompressible 
fluid flow is rewritten as follows

$$
\begin{aligned}
& \frac{\partial \phi_{\theta \theta}(\boldsymbol{X}, \boldsymbol{\xi}, t)}{\partial t}+\frac{1}{2}\left(\left\langle u_{j A}\right\rangle+\left\langle u_{j B}\right\rangle\right)(\boldsymbol{X}, \boldsymbol{\xi}, t) \frac{\partial \phi_{\theta \theta}(\boldsymbol{X}, \boldsymbol{\xi}, t)}{\partial X_{j}}=-\phi_{j \theta}(\boldsymbol{X}, \boldsymbol{\xi}, t) \frac{\partial\left\langle\theta_{A}\right\rangle}{\partial x_{j A}} \\
& =-\phi_{\theta j}(\boldsymbol{X}, \boldsymbol{\xi}, t) \frac{\partial\left\langle\theta_{B}\right\rangle}{\partial x_{j B}}-\left(\left\langle u_{j B}\right\rangle-\left\langle u_{j A}\right\rangle\right)(\boldsymbol{X}, \boldsymbol{\xi}, t) \frac{\partial \phi_{\theta \theta}(\boldsymbol{X}, \boldsymbol{\xi}, t)}{\partial \xi_{j}} \\
& -\frac{1}{2} \frac{\partial}{\partial X_{j}}\left(\left\langle\theta_{A}^{\prime} u_{j A}^{\prime} \theta_{B}^{\prime}\right\rangle+\left\langle\theta_{A}^{\prime} u_{j B}^{\prime} \theta_{B}^{\prime}\right\rangle\right)(\boldsymbol{X}, \boldsymbol{\xi}, t) \\
& -\frac{\partial}{\partial \xi_{j}}\left(\left\langle\theta_{A}^{\prime} u_{j B}^{\prime} \theta_{B}^{\prime}\right\rangle-\left\langle\theta_{A}^{\prime} u_{j A}^{\prime} \theta_{B}^{\prime}\right\rangle\right)(\boldsymbol{X}, \boldsymbol{\xi}, t) \\
& +\frac{\sigma}{2} \frac{\partial^{2} \phi_{\theta \theta}}{\partial X_{j} \partial X_{j}}(\boldsymbol{X}, \boldsymbol{\xi}, t)+2 \sigma \frac{\partial^{2} \phi_{\theta \theta}}{\partial \xi_{j} \partial \xi_{j}}(\boldsymbol{X}, \boldsymbol{\xi}, t)
\end{aligned}
$$

Before taking the Fourier transform of this equation, it is convenient to introduce Taylor series expansion in space of the terms involving the velocities leading to

$$
\begin{gathered}
\left(\left\langle u_{k B}\right\rangle-\left\langle u_{k A}\right\rangle\right)(\boldsymbol{X}, \boldsymbol{\xi})=\xi_{m} \frac{\partial\left\langle u_{k}\right\rangle}{\partial X_{m}}(\boldsymbol{X})+O\left(\xi^{2}\right) \\
\left(\left\langle u_{k A}\right\rangle+\left\langle u_{k B}\right\rangle\right)(\boldsymbol{X}, \boldsymbol{\xi})=2\left\langle u_{k}\right\rangle(\boldsymbol{X})+\frac{\xi_{m} \xi_{p}}{2} \frac{\partial^{2}\left\langle u_{k}\right\rangle}{\partial X_{m} \partial X_{p}}(\boldsymbol{X})+O\left(\xi^{3}\right),
\end{gathered}
$$

It is also useful to develop the production term involving the derivative of the mean temperature as follows

$$
\phi_{j \theta} \frac{\partial\left\langle\theta_{A}\right\rangle}{\partial x_{j A}}+\phi_{\theta j} \frac{\partial\left\langle\theta_{B}\right\rangle}{\partial x_{j B}}=\phi_{j \theta} \frac{\partial\langle\theta\rangle}{\partial X_{j}}+\phi_{\theta j} \frac{\partial\langle\theta\rangle}{\partial X_{j}}-\frac{\xi_{m}}{2} \frac{\partial^{2}\langle\theta\rangle}{\partial X_{m} \partial X_{j}}\left(\phi_{j \theta}-\phi_{\theta j}\right)+O\left(\xi^{2}\right)
$$

Here, we restrict the development only to its first term to get a tractable equation. This means that the Fourier terms are identical to the ones in homogeneous anisotropic turbulence. Using Eqs. (66), (67) and (68), Eq. (65) including contributions of homogeneous and nonhomogeneous terms becomes

$$
\begin{aligned}
& \frac{\partial \phi_{\theta \theta}(\boldsymbol{X}, \boldsymbol{\xi}, t)}{\partial t}+\left\langle u_{j}\right\rangle(\boldsymbol{X}) \frac{\partial \phi_{\theta \theta}(\boldsymbol{X}, \boldsymbol{\xi}, t)}{\partial X_{j}} \\
& =-\phi_{j \theta}(\boldsymbol{X}, \boldsymbol{\xi}, t) \frac{\partial\left\langle\theta_{A}\right\rangle}{\partial X_{j}}-\phi_{\theta j}(\boldsymbol{X}, \boldsymbol{\xi}, t) \frac{\partial\left\langle\theta_{B}\right\rangle}{\partial X_{j}} \\
& -\xi_{m} \frac{\partial\left\langle u_{j}\right\rangle}{\partial X_{m}} \frac{\partial \phi_{\theta \theta}}{\partial \xi_{j}}(\boldsymbol{X}, \boldsymbol{\xi}, t)-\frac{1}{2} \frac{\partial}{\partial X_{j}}\left(\left\langle\theta_{A}^{\prime} u_{j A}^{\prime} \theta_{B}^{\prime}\right\rangle+\left\langle\theta_{A}^{\prime} u_{j B}^{\prime} \theta_{B}^{\prime}\right\rangle\right)(\boldsymbol{X}, \boldsymbol{\xi}, t) \\
& -\frac{\partial}{\partial \xi_{j}}\left(\left\langle\theta_{A}^{\prime} u_{j B}^{\prime} \theta_{B}^{\prime}\right\rangle-\left\langle\theta_{A}^{\prime} u_{j A}^{\prime} \theta_{B}^{\prime}\right\rangle\right)(\boldsymbol{X}, \boldsymbol{\xi}, t)+\frac{\sigma}{2} \frac{\partial^{2} \phi_{\theta \theta}}{\partial X_{j} \partial X_{j}}(\boldsymbol{X}, \boldsymbol{\xi}, t) \\
& +2 \sigma \frac{\partial^{2} \phi_{\theta \theta}}{\partial \xi_{j} \partial \xi_{j}}(\boldsymbol{X}, \boldsymbol{\xi}, t)
\end{aligned}
$$


The nonhomogeneous terms that appear in Eq. (69) correspond to the usual terms in one-point equation whereas the other terms involving the distance $\xi$ can be treated as in homogeneous anisotropic turbulence. So, this method can be viewed as considering the tangent homogeneous anisotropic field at the point $\boldsymbol{X}$ of the nonhomogeneous field.

\section{A.2 Transport equation of the Fourier transform of the two-point corre- lation tensor}

Considering the Fourier transform of $\phi_{\theta \theta}(\boldsymbol{X}, \boldsymbol{\xi}, t)$ that is expressed as

$$
\widehat{\phi_{\theta \theta}}(\boldsymbol{X}, \boldsymbol{\kappa}, t)=\int_{-\infty}^{\infty} \int_{-\infty}^{\infty} \int_{-\infty}^{\infty} \phi_{\theta \theta}(\boldsymbol{X}, \boldsymbol{\xi}, t) \exp (-j \boldsymbol{\kappa} \boldsymbol{\xi}) d \boldsymbol{\xi}
$$

the Fourier transform of the transport equation of the double velocity correlation in locally tangent spectral space then reads

$$
\begin{aligned}
& \frac{\partial \widehat{\phi_{\theta \theta}}(\boldsymbol{X}, \boldsymbol{\kappa}, t)}{\partial t}+\left\langle u_{j}\right\rangle(\boldsymbol{X}) \frac{\partial \widehat{\phi_{\theta \theta}}(\boldsymbol{X}, \boldsymbol{\kappa}, t)}{\partial X_{j}} \\
& =-\widehat{\phi_{j \theta}}(\boldsymbol{X}, \boldsymbol{\kappa}, t) \frac{\partial\left\langle\theta_{A}\right\rangle}{\partial X_{j}}-\widehat{\phi_{\theta j}}(\boldsymbol{X}, \boldsymbol{\kappa}, t) \frac{\partial\left\langle\theta_{B}\right\rangle}{\partial X_{j}} \\
& +\kappa_{j} \frac{\partial\left\langle u_{j}\right\rangle}{\partial X_{m}} \frac{\partial \phi_{\theta \theta}}{\partial \kappa_{m}}(\boldsymbol{X}, \boldsymbol{\kappa}, t)-\frac{1}{2} \frac{\partial}{\partial X_{j}}\left(S_{\theta j, \theta}+S_{\theta, j \theta}\right)-j \kappa_{j}\left(S_{\theta, j \theta}-S_{\theta j, \theta}\right) \\
& +\frac{\sigma}{2} \frac{\partial^{2} \widehat{\phi_{\theta \theta}}}{\partial X_{j} \partial X_{j}}(\boldsymbol{X}, \boldsymbol{\kappa}, t)-2 \sigma \kappa^{2} \widehat{\phi_{\theta \theta}}(\boldsymbol{X}, \boldsymbol{\kappa}, t)
\end{aligned}
$$

where $S_{\theta j, \theta}=\left\langle\theta_{A}^{\prime} u_{j A}^{\prime} \theta_{B}^{\prime}\right\rangle$ and $S_{\theta, j \theta}=\left\langle\theta_{A}^{\prime} u_{j B}^{\prime} \theta_{B}^{\prime}\right\rangle$.

\section{A.3 Transport equation of the spherical mean of the Fourier transform of the two-point correlation tensor}

Considering $\phi_{\theta \theta}(\boldsymbol{X}, t)$ and its Fourier transform $\widehat{\phi_{\theta \theta}}(\boldsymbol{X}, \boldsymbol{\kappa}, t)$, we define the spherical mean of the Fourier transform by the relation

$$
\varphi_{\theta \theta}(\boldsymbol{X}, \kappa, t)=\left[\phi_{\theta \theta}(\boldsymbol{X})\right]^{\Delta}(\kappa, t)=\frac{1}{4 \pi \kappa^{2}} \int_{0}^{\pi} \int_{0}^{2 \pi} \widehat{\phi_{\theta \theta}}(\boldsymbol{X}, \boldsymbol{\kappa}, t) \kappa^{2} \sin \theta d \theta d \phi
$$


The transport equation of the one-dimensional spectral tensor of the double temperature correlations is obtained by taking the Fourier transform and mean integration over spherical shells of Eq. (71). As mentioned in Refs. [15, 4], spherical averages allow to make some useful simplifications in the spectral equations. The directional information is lost but the spectral averaged correlation of the fluctuating temperatures is then only function of the wavenumber and not anymore of the wavevector. For sake of clarity, we denote $E_{\theta}=\varphi_{\theta \theta} / 2$. In this case, the mistress transport equation for $E_{\theta}$ then reads

$$
\begin{aligned}
& \frac{\partial E_{\theta}}{\partial t}(\boldsymbol{X}, \kappa, t)+\left\langle u_{k}\right\rangle(\boldsymbol{X}) \frac{\partial E_{\theta}(\boldsymbol{X}, \kappa, t)}{\partial X_{k}} \\
& =\mathcal{P}_{\theta}(\boldsymbol{X}, \kappa, t)+\mathcal{T}_{\theta}(\boldsymbol{X}, \kappa, t)+\mathcal{J}_{\theta}(\boldsymbol{X}, \kappa, t)-\mathcal{E}_{\theta}(\boldsymbol{X}, \kappa, t)
\end{aligned}
$$

where on the right hand side of Eq. (73), $\mathcal{P}_{\theta j}$ is the production term defined by

$$
\mathcal{P}_{\theta}(\boldsymbol{X}, \kappa, t)=-\varphi_{j \theta}(\boldsymbol{X}, \kappa, t) \frac{\partial\langle\theta\rangle}{\partial X_{j}}
$$

$\mathcal{T}_{\theta}$ is the transfer term including two different contributions of different meaning

$$
\begin{aligned}
& \mathcal{T}_{\theta}(\boldsymbol{X}, \kappa, t)=-\frac{1}{2}\left(\xi_{m} \frac{\partial \phi_{\theta \theta}}{\partial \xi_{j}}(\boldsymbol{X}, \boldsymbol{\xi}, t)\right)^{\Delta} \frac{\partial\left\langle u_{j}\right\rangle}{\partial X_{m}} \\
& -\frac{1}{2}\left(\frac{\partial}{\partial \xi_{j}}\left(S_{\theta, j \theta}-S_{\theta j, \theta}(\boldsymbol{X}, \boldsymbol{\xi}, t)\right)\right)^{\Delta}
\end{aligned}
$$

$\mathcal{J}_{\theta}$ denotes the diffusion term given by

$$
\mathcal{J}_{\theta}(\boldsymbol{X}, \kappa, t)=-\frac{1}{4} \frac{\partial}{\partial X_{j}}\left(S_{\theta, j \theta}^{\Delta}+S_{\theta j, \theta}^{\Delta}\right)+\sigma \frac{\partial^{2} E_{\theta}}{\partial X_{j} \partial X_{j}}(\boldsymbol{X}, \boldsymbol{\xi}, t)
$$

and finally, $\mathcal{E}_{\theta}$ is the dissipation rate including two different contributions as

$$
\mathcal{E}_{\theta}=\frac{\sigma}{2} \frac{\partial^{2} E_{\theta}}{\partial X_{j} \partial X_{j}}(\boldsymbol{X}, \boldsymbol{\xi}, t)+2 \sigma \kappa^{2} E_{\theta}(\boldsymbol{X}, \boldsymbol{\xi}, t)
$$

Indeed, from the derivatives formula, it is straightforward to get

$$
\left[\frac{\partial^{2} \phi_{\theta \theta}\left(\boldsymbol{x}_{A}, \boldsymbol{x}_{B}\right)}{\partial x_{j A} \partial x_{j B}}\right]^{\Delta}=\frac{1}{4} \frac{\partial^{2} \varphi_{\theta \theta}}{\partial X_{j} \partial X_{j}}(\boldsymbol{X}, \boldsymbol{\xi})-\frac{\partial^{2} \varphi_{\theta \theta}}{\partial \xi_{j} \partial \xi_{j}}(\boldsymbol{X}, \boldsymbol{\xi})
$$

showing the direct physical interpretation of the scalar dissipation term. As a result of interest, it is simple to see that the full integration in the spectral space of $\mathcal{E}_{\theta}$ when $\boldsymbol{\xi}$ goes to zero allows to recover the usual dissipation-rate $\epsilon_{\theta}$

$$
\epsilon_{\theta}=\int_{0}^{\infty}\left[\frac{\sigma}{2} \frac{\partial^{2} E_{\theta \theta}}{\partial X_{j} \partial X_{j}}+2 \sigma \kappa_{j}^{2} E_{\vartheta \vartheta}\right]_{\xi=0} d \kappa
$$


so that, using the partial derivative operators defined in Appendix A (Chaouat and Schiestel, 2007)

$$
\epsilon_{\theta}=\frac{\sigma}{4}\left[\frac{\partial^{2} \phi_{\theta \theta}}{\partial X_{j} \partial X_{j}}\right]_{\xi=0}-\sigma\left[\frac{\partial^{2} \phi_{\theta \theta}}{\partial \xi_{j} \partial \xi_{j}}\right]_{\xi=0}=\sigma\left\langle\frac{\partial \theta^{\prime}}{\partial x_{j}} \frac{\partial \theta^{\prime}}{\partial x_{j}}\right\rangle
$$

\section{B INFLUENCE OF PRANDTL NUMBER IN THE SCALAR DISSIPATION EQUATION}

\section{B.1 The case of molecular Prandtl numbers near unity}

The scalar variance $k_{\theta}$ is obtained by integrating the spectrum (37) from 0 to infinity leading to

$$
k_{\theta}=\int_{0}^{\infty} \frac{C_{\theta} \epsilon_{\theta}}{C_{K} \epsilon} E(\kappa) d \kappa=\frac{C_{\theta} \epsilon_{\theta} k}{C_{K} \epsilon}
$$

So that the reduced spectrum $E_{\theta}^{*}(\vartheta)$ defined by $E_{\theta}^{*}(\vartheta)=E_{\theta}(\kappa) / k_{\theta} L$ takes the simple expression written as $E_{\theta}^{*}(\vartheta)=E(\kappa) / k L$ or equivalently $E_{\theta}^{*}(\vartheta)=E^{*}(\vartheta)$. One can then derive the practical formulation of the dissipation equation of scalar variance for molecular Prandtl numbers near unity corresponding to Fig. 2. Using Eq. (37), it is then simple matter to compute the subfilter part of the variance $k_{\theta s f s}$ with the change of variable $\vartheta=\kappa L$ by

$$
\frac{k_{\theta s f s}}{k_{\theta}}=\int_{\vartheta_{c}}^{\infty} E_{\theta}^{*}(\vartheta) d \vartheta=\int_{\vartheta_{c}}^{\infty} E^{*}(\vartheta) d \vartheta
$$

Using the energy spectrum defined in Refs. (Chaouat and Schiestel, 2009; Chaouat, 2017)

$$
E^{*}(\vartheta)=\frac{\frac{2}{3} \beta \vartheta^{\alpha-1}}{\left[1+\beta \vartheta^{\alpha}\right]^{\gamma+1}}
$$

where $\alpha$ and $\beta$ are constant coefficients, one gets $c_{\epsilon_{\theta \theta_{2} s f s}}$ as

$$
c_{\epsilon_{\theta \theta_{2} s f s}}=c_{\epsilon_{\theta \theta_{1}}}+\frac{\Delta c_{\epsilon_{\theta \theta}}}{\left[1+\beta \vartheta_{c}^{\alpha}\right]^{\gamma}}
$$

where $\Delta c_{\epsilon_{\theta \theta}}=c_{\epsilon_{\theta \theta_{2}}}-c_{\epsilon_{\theta \theta_{1}}}$. As expected, the spectral properties of the scalar variance at Prandtl numbers near unity is very similar to the ones for velocity variance, expressing some Reynolds analogy principle. However, when the Prandtl number is distinctly different from unity, the spectral properties become different and new spectral laws are involved. In this respect, for the following developments, we shall rely upon the well known studies of Batchelor (1959), Batchelor et al. (1959) and Obukhov (1959), and reinterpreted afterwards by Warhaft (2000). 


\section{B.2 The case of small molecular Prandtl numbers}

Each contribution $k_{\theta s f s}$ and $k_{\theta}$ can be computed by integrating the spectrum $E(\kappa)$ from their respective wave number ranges $\left[\kappa_{c}, \kappa_{e}\right]$ and $\left[0, \kappa_{e}\right]$. The first point is to calculate $k_{\theta}$ using Eq. (41). As a result, it is a simple matter to obtain

$$
k_{\theta}=\int_{0}^{\infty} E_{\theta}(\kappa) d \kappa=\frac{C_{\theta} \epsilon_{\theta} k}{C_{K} \epsilon} \int_{0}^{\vartheta_{H}} E^{*}(\vartheta) d \vartheta=\frac{C_{\theta} \epsilon_{\theta} k}{C_{K} \epsilon}\left(1-\left[1+\beta \vartheta_{H}^{\alpha}\right]^{-\gamma}\right)
$$

so that the spectrum $E_{\theta}(\kappa)$ given by Eq. (41) can be rewritten as

$$
E_{\theta}(\kappa)=\frac{k_{\theta}}{k} \frac{E(\kappa) H\left(\kappa_{H}-\kappa\right)}{\left(1-\left[1+\beta \vartheta_{H}^{\alpha}\right]^{-\gamma}\right)}
$$

In the first case where $\vartheta_{c}<\vartheta_{H}$, the ratio $k_{\theta s f s} / k_{\theta}$ can be computed easily using the spectrum given by Eq. (86) as

$$
\frac{k_{\theta s f s}}{k_{\theta}}=\int_{\vartheta_{c}}^{\vartheta_{H}} E_{\theta}^{*}(\vartheta) d \vartheta
$$

leading to the result

$$
\frac{k_{\theta s f s}}{k_{\theta}}=\frac{\left[1+\beta \vartheta_{c}^{\alpha}\right]^{-\gamma}-\left[1+\beta P e_{t}^{3 \alpha / 4}\right]^{-\gamma}}{1-\left[1+\beta P e_{t}^{3 \alpha / 4}\right]^{-\gamma}}
$$

In the second case where $\vartheta_{c}>\vartheta_{H}$, then $k_{\theta s f s} / k_{\theta}=0$, but, in this case, there is no longer need to solve the $\epsilon_{\theta}$ equation! The exact expression of the coefficient $c_{\epsilon_{\theta \theta_{2}} s f s}$ is then

$$
c_{\epsilon_{\theta \theta_{2} s f s}}= \begin{cases}c_{\epsilon_{\theta \theta_{1}}}+\Delta c_{\epsilon_{\theta \theta}} \frac{\left[1+\beta \vartheta_{c}^{\alpha}\right]^{-\gamma}-\left[1+\beta P e_{t}^{3 \alpha / 4}\right]^{-\gamma}}{1-\left[1+\beta P e_{t}^{3 \alpha / 4}\right]^{-\gamma}} & \left(\vartheta_{c}<\vartheta_{H}\right) \\ c_{\epsilon_{\theta \theta_{1}}} & \left(\vartheta_{c}>\vartheta_{H}\right)\end{cases}
$$

\section{B.3 The case of large molecular Prandtl numbers}

The integration of $E_{\theta}(\kappa)$ over the wave number range is given by

$$
k_{\theta}=\int_{0}^{\kappa_{K}} E_{\theta_{1}}(\kappa) d \kappa+\int_{\kappa_{K}}^{\infty} E_{\theta_{2}}(\kappa) d \kappa
$$


In the first case where $\vartheta_{c}<\vartheta_{K}$, then

$$
\frac{k_{\theta s f s}}{k_{\theta}}=\int_{\vartheta_{c}}^{\vartheta_{K}} E_{\vartheta_{1}}^{*}(\vartheta) d \vartheta+\int_{\vartheta_{K}}^{\vartheta_{S}} E_{\vartheta_{2}}^{*}(\vartheta) d \vartheta
$$

Noticing that

$$
\frac{\vartheta_{S}}{\vartheta_{K}}=\frac{\kappa_{S}}{\kappa_{K}}=\frac{\eta_{K}}{\eta_{\theta}^{*}}=\operatorname{Pr}^{1 / 2}
$$

Eq. (91) leads to

$$
\frac{k_{\theta s f s}}{k_{\theta}}=\frac{\left[1+\beta \vartheta_{c}^{\alpha}\right]^{-\gamma}-\left[1+\beta R e_{t}^{3 \alpha / 4}\right]^{-\gamma}+c_{\theta} \frac{\epsilon_{\theta}}{k_{\theta}}\left(\frac{\nu}{\epsilon}\right)^{1 / 2} \ln P_{r}^{1 / 2}}{1-\left[1+\beta R e_{t}^{3 \alpha / 4}\right]^{-\gamma}+c_{\theta} \frac{\epsilon_{\theta}}{k_{\theta}}\left(\frac{\nu}{\epsilon}\right)^{1 / 2} \ln P_{r}^{1 / 2}}
$$

In the second case where $\vartheta_{K}<\vartheta_{c}<\vartheta_{S}$, then

$$
\frac{k_{\theta s f s}}{k_{\theta}}=\int_{\vartheta_{c}}^{\vartheta_{S}} E_{\vartheta_{2}}^{*}(\vartheta) d \vartheta
$$

yielding

$$
\frac{k_{\theta_{s f s}}}{k_{\theta}}=\frac{c_{\theta} \frac{\epsilon_{\theta}}{k_{\theta}}\left(\frac{\nu}{\epsilon}\right)^{1 / 2} \ln \frac{\vartheta_{S}}{\vartheta_{c}}}{1-\left[1+\beta R e_{t}^{3 \alpha / 4}\right]^{-\gamma}+c_{\theta} \frac{\epsilon_{\theta}}{k_{\theta}}\left(\frac{\nu}{\epsilon}\right)^{1 / 2} \ln P_{r}^{1 / 2}}
$$

In the third case where $\vartheta_{S}<\vartheta_{c}$, then $k_{\theta s f s} / k_{\theta}=0$. As a result, we obtain the exact expression for the coefficient $c_{\epsilon_{\theta \theta_{2} s f s}}$ valid in all cases

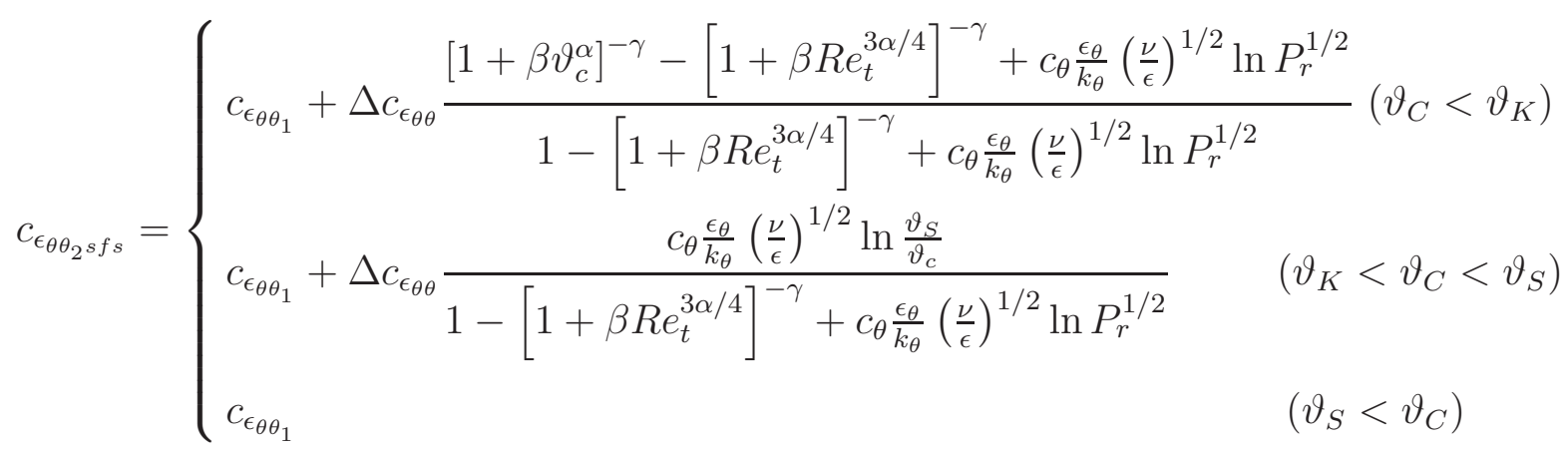

\section{THE VISCOUS SUBLAYER LIMIT}

The low Reynolds number extension of the model equations for the velocities and stresses have been defined in previous papers (Chaouat and Schiestel, 2005, 2013). In the present case, Eq. (54) for 
the variance dissipation-rate $\epsilon_{\theta}$ is modeled at low Reynolds number to approach walls using the same empirical approach that is of practical use for simulation of confined flows. As a result of modeling, the transport equation for $\epsilon_{\theta}$ reads

$$
\frac{\partial \epsilon_{\theta}}{\partial t}=c_{\epsilon_{\theta \theta_{1} s f s}} P_{\theta s f s} \frac{\epsilon_{\theta}}{k_{\theta s f s}}+c_{\epsilon_{\theta k_{1} s f s}} f_{\epsilon_{\theta k_{1}}} P_{s f s} \frac{\epsilon_{\theta}}{k_{s f s}}-c_{\epsilon_{\theta k_{2} s f s}} \frac{\epsilon_{\theta} \tilde{\epsilon}}{k_{s f s}}-c_{\epsilon_{\theta \theta_{2} s f s}} \frac{\epsilon_{\theta} \tilde{\epsilon}_{\theta}}{k_{\theta s f s}}+J_{\epsilon_{\theta}}
$$

where $\tilde{\epsilon}=\epsilon-2 \nu\left[\partial\left(k_{s f s}^{1 / 2}\right) / \partial x_{n}\right]^{2}, \tilde{\epsilon_{\theta}}=\epsilon_{\theta}-2 \sigma\left[\partial\left(k_{\theta f f s}^{1 / 2}\right) / \partial x_{n}\right]^{2}$ and $f_{\epsilon_{\theta k_{1}}}$ is a damping function of the Prandtl and Reynolds numbers which has been calibrated as $f_{\epsilon_{\theta k_{1}}}=0.4\left(1-P_{r}\right) /\left(1+P_{r}\right) \exp \left[-\left(\sqrt{\operatorname{Re}_{s f s}} / 100\right)\right]$ where $R e_{s f s}=k_{s f s}^{2} /\left(\nu \epsilon_{s f s}\right)$. This formulation at low Reynolds number as a function of the Prandtl number is inspired from previous papers, see for instance Shikazono and Kasagi (1996); Nagano and Shimada (1996); Kenjeres and Hanjalic (2000). Taylor series expansion in space for the fluctuating velocities $u_{i}^{\prime}$ and fluctuating scalar variable $\theta^{\prime}$ associated with the passive scalar show that the ratios $\tilde{\epsilon} / k_{s f s}$ and $\tilde{\epsilon}_{\theta} / k_{\theta s f s}$ take finite values at the wall for $x_{n}=0$. In particular, the boundary condition for $\epsilon$ and $\epsilon_{\theta}$ at the wall are given by the relations $\tilde{\epsilon}_{w}=2 \nu\left[\partial\left(k_{s f s}^{1 / 2}\right) / \partial x_{n}\right]_{w}^{2}$ and $\tilde{\epsilon}_{\theta w}=2 \sigma\left[\partial\left(k_{\theta_{s f s}}^{1 / 2}\right) / \partial x_{n}\right]_{w}^{2}$. The code developed by Chaouat (2011) is based on the finite volume technique so that the equations are solved in the grid cell around the grid-point of the mesh and not on the grid point itself. 


\section{References}

[1] Batchelor, G. K., 1959. Small scale variation of convected quantities like temperature in a turbulent fluid. Part 1. General discussion and the case of small conductivity. J. Fluid Mech. 5, 113-133.

[2] Batchelor, G. K., Howells, I. D., Townsend, A., 1959. Small-scale variation of convected quantities like temperature in turbulent fluid. Part 2: The case of large conductivity. J. Fluid Mech. 5, 134-139.

[3] Briard, A., Gomez, T., Cambon, C., 2016. Spectral modelling for passive scalar dynamics in homogeneous anisotropic turbulence. J. Fluid Mech. 799, 159-199.

[4] Cambon, C., Jeandel, D., Mathieu, J., 1981. Spectral modelling of homogeneous non-isotropic turbulence. J. Fluid Mech. 104, 247-262.

[5] Chaouat, B., 2010. Subfilter scale transport model for hybrid RANS/LES simulations applied to a complex bounded flow. J. Turbul. 11 (51), 1-30.

[6] Chaouat, B., 2011. An efficient numerical method for RANS/LES turbulent simulations using subfilter scale stress transport equations. Int. J. Numer. Methods Fluids 67, 1207-1233.

[7] Chaouat, B., 2012. Simulation of turbulent rotating flows using a subfilter scale stress model derived from the partially integrated transport modeling method. Phys. Fluids 24, 045108, 1-35.

[8] Chaouat, B., 2017. The state of the art of hybrid RANS/LES modeling for the simulation of turbulent flows. Flow, Turbul. Combust. 99, 279-327.

[9] Chaouat, B., 2017b. Application of the PITM method using inlet synthetic turbulence generation for the simulation of the turbulent flow in a small axisymmetric contraction. Flow, Turbul. Combust. 98, 987-1024.

[10] Chaouat, B., 2017c. Commutation errors in PITM simulations. Int. J. Heat Fluid Flow 67, 138-154.

[11] Chaouat, B., 2018. DNS of passive scalar transport fields in turbulent flow at low and high Prandtl numbers. In Proceedings of the 12th International ERCOFTAC Symposium on Enginnering Turbulence Modelling and Measurements, Montpellier, France, S1, 1-6. 
[12] Chaouat, B., Peyret, C., 2019. Investigation of the wall scalar fluctuations effect on passive scalar turbulent fields at several Prandtl numbers by means of direct numerical simulations. J. Heat Transfer, ASME 141, 1-9.

[13] Chaouat, B., Schiestel, R., 2002. Reynolds stress transport modelling for steady and unsteady channel flows with wall injection. J. Turbul. 3, 1-15.

[14] Chaouat, B., Schiestel, R., 2005. A new partially integrated transport model for subgrid-scale stresses and dissipation rate for turbulent developing flows. Phys. Fluids 17, 065106, 1-19.

[15] Chaouat, B., Schiestel, R., 2007. From single-scale turbulence models to multiple-scale and subgridscale models by Fourier transform. Theoret. Comput. Fluid Dyn. 21, 201-229.

[16] Chaouat, B., Schiestel, R., 2009. Progress in subgrid-scale transport modelling for continuous hybrid non-zonal RANS/LES simulations. Int. J. Heat Fluid Flow 30, 602-616.

[17] Chaouat, B., Schiestel, R., 2012. Analytical insights into the partially integrated transport modeling method for hybrid Reynolds averaged Navier-Stokes equations-large eddy simulations of turbulent flows. Phys. Fluids 24, 085106, 1-34.

[18] Chaouat, B., Schiestel, R., 2013a. Partially integrated transport modeling method for turbulence simulation with variable filters. Phys. Fluids 25, 125102, 1-39.

[19] Chaouat, B., Schiestel, R., 2013b. Hybrid RANS-LES simulations of the turbulent flow over periodic hills at high Reynolds number using the PITM method. Comput. Fluids 84, 279-300.

[20] Corrsin, S., 1964. Further generalizations of Onsager's cascade model for turbulent spectra. Phys. Fluids 7, 1156-1159.

[21] Daly, B. J., Harlow, F. H., 1970. Transport equations in turbulence. Phys. Fluids. 13, 11, 2634-2649.

[22] Favre, A., Kovasznay, L. S. G., Dumas, R., Gaviglio, J., Coantic, M., 1976. La turbulence en mécanique des fluides. Gauthiers-Villars. 
[23] Foroutan, H., Yavuzkurt, S., 2014. A partially averaged Navier Stokes model for the simulation of turbulent swirling flow with vortex breakdown. Int. J. Heat Fluid Flow 50, 402-416.

[24] Fröhlich J., Von Terzi, D., 2008. Hybrid LES/RANS methods for the simulation of turbulent flows. Prog. Aerosp. Sci. 44, 349-377.

[25] Gatski, T. B., 2009. Second-moment and scalar flux representations in engineering and geophysical flows. Fluid Dyn. Res. 41, 012202, 1-24.

[26] Gharbi, A., Ruffin, E., Anselmet, F., Schiestel, R., 1996. Numerical modelling of variable density turbulent jets. Int. J. Heat Mass Transfer 39, 1865-1882.

[27] Hanjalic, K., Launder, B. E., 2011. Modelling turbulence in engineering and the environment. Second-moment route to closure. Cambridge University Press.

[28] Heinz, S., 2019. The large eddy simulation capability of Reynolds-averaged Navier-Stokes equations: Analytical results. Phys. Fluids 31, 021702, 1-6.

[29] Hinze, J. O., 1975. Turbulence, (Mc Graw-Hill, New York.

[30] Jeandel D., Brison, J.F., Mathieu, J., 1978. Modeling methods in physical and spectral spaces. Phys. Fluids 100, 21, 169-181.

[31] Jones, W. P., Musonge, P., 1988. Closure of the Reynolds stress and scalar flux equations. Phys. Fluids 31, 3589-3604.

[32] Kenjeres, S., Hanjalic, K., 2006. LES,T-RANS and hybrid simulations of thermal convection at high Ra number. Int. J. Heat Fluid Flow 27, 800-810.

[33] Kenjeres, S., Hanjalic, K., 2000. Convective rolls and heat transfer in finite-length Rayleigh-Benard convection: A two-dimensional numerical study. Phys. Review E 62, 7987-7998.

[34] Kenjeres, S., Gunarjo, S. B., Hanjalic, K., 2005. Contribution to elliptic relaxation modelling of turbulent natural and mixed convection. Int. J. Heat Fluid Flow 26, 569-586. 
[35] Kenjeres, S., de Wildt, S., Busking, T., 2015. Capturing transient effects in turbulent flows over complex urban areas with passive pollutants. Int. J. Heat Fluid Flow 51, 120-137.

[36] Launder, B. E., 1988. On the computation of convective heat transfer in complex turbulent flows. J. Heat Transfer 88, 1112-1128.

[37] Launder, B. E., Reece, G. J., Rodi, W., 1975. Progress in the development of a Reynolds stress turbulence closure. J. Fluid Mech. 101, 68, 537.

[38] Launder, B. E., Reynolds, W. C., Rodi, W., Mathieu, J., Jeandel, D., 1984. Simulation of turbulence models and their applications, Vol. 2, Eyrolles.

[39] Mathieu, J., Jeandel, D., Launder, B. E., Reynolds, W. C., Rodi, W., 1894. Simulation of turbulence models and their applications. Vol. 1, Eyrolles.

[40] Mishra, A. A., Girimaji, S. S., 2017. Towards approximating non-local dynamics in single-point pressure strain correlation closures. J. Fluid Mech. 811, 168-188.

[41] Monin, A. S., Yaglom, A. M., 1975. Statistical Fluid Mechanics. The M.I.T. Press Cambridge, Massachusetts, vols. I and II.

[42] Mons, V., Cambon, C., Sagaut, P., 2016. A spectral model for homogeneous shear-driven anisotropic turbulence in terms of spherically averaged descriptors. J. Fluid Mech. 788, 147-182.

[43] Nagano, Y., Kim, C., 1988. A two-equation model for heat transport in wall turbulent shear flows. J. Heat Transfer 110, 583-589.

[44] Nagano, Y., Shimada, M., 1996. Development of a two-equation heat transfer model based on direct simulations of turbulent flows with different Prandtl numbers. Phys. Fluids 8, 3379-3402.

[45] Newmann, G. R., Launder, B. E., Lumley, J. L., 1981. Modelling the behaviour of homogeneous scalar turbulence. J. Fluid Mech. 111, 217-232.

[46] Oboukhov, A. M., 1959. Structure of the temperature field in turbulent flows. Izvestiia Akademii Nauk SSSR, Ser Geogr. i Geofiz. 91, 58-69. 
[47] Ruffin, E., Schiestel, R., Anselmet, F., Amielh, M., Fulachier, L., 1994. Investigation of characteristic scales in variable density turbulent jets using a second-order model. Phys. Fluids 6, 2785-2799.

[48] Schiestel, R., 1983a. Sur le concept d'échelles multiples en modélisation des écoulements turbulents, Part I. J. Theor. Appl. Mech. 2, 3, 417-449.

[49] Schiestel, R., 1983b. Sur le concept d'échelles multiples en modélisation des écoulements turbulents, Part II. J. Theor. Appl. Mech. 2, 4, 601-628.

[50] Schiestel, R., 1987. Multiple-time scale modeling of turbulent flows in one point closures. Phys. Fluids 30, 722-731.

[51] Schiestel, R., 2008. Modeling and simulation of turbulent flows. ISTE Ltd and J. Wiley.

[52] Schiestel, R., Dejoan, A., 2005. Towards a new partially integrated transport model for coarse grid and unsteady turbulent flow simulations. Theoret. Comput. Fluid Dyn. 18, 443-468.

[53] Shikazono, N., Kasagi, N., 1996. Second-moment closure for turbulent scalar transport at various Prandtl numbers. Int. J. Heat Mass Transfer 39, 2977-2987.

[54] Suga, K., 2004. Improvement of second-moment closure for turbulent obstacle flow and heat transfer. Int. J. Heat Fluid Flow 25, 776-784.

[55] Tennekes, H., Lumley, J. L., 1972. A first course of turbulence, The M.I.T. Press Cambridge, Massachusetts.

[56] Warhaft, Z., 2000. Passive scalars in turbulent flows. Annu. Rev. Fluid Mech. 32, 203-240.

[57] Yoshizawa, A., 1982. A statistically-derived subgrid model for the large-eddy simulation of turbulence. Phys. Fluids 25, 1532-1538.

[58] Yoshizawa, A., 1988. Statistical modelling of passive-scalar diffusion in turbulent shear flows. J. Fluid Mech. 195, 541-555. 